\title{
Exoplanet Atmosphere Measurements from Direct Imaging
}

\author{
Beth A. Biller and Mickaël Bonnefoy
}

\begin{abstract}
In the last decade, about a dozen giant exoplanets have been directly imaged in the IR as companions to young stars. With photometry and spectroscopy of these planets in hand from new extreme coronagraphic instruments such as SPHERE at VLT and GPI at Gemini, we are beginning to characterize and classify the atmospheres of these objects. Initially, it was assumed that young planets would be similar to field brown dwarfs, more massive objects that nonetheless share similar effective temperatures and compositions. Surprisingly, young planets appear considerably redder than field brown dwarfs, likely a result of their low surface gravities and indicating much different atmospheric structures. Preliminarily, young free-floating planets appear to be as or more variable than field brown dwarfs, due to rotational modulation of inhomogeneous surface features. Eventually, such inhomogeneity will allow the top of atmosphere structure of these objects to be mapped via Doppler imaging on extremely large telescopes. Direct imaging spectroscopy of giant exoplanets now is a prelude for the study of habitable zone planets. Eventual direct imaging spectroscopy of a large sample of habitable zone planets with future telescopes such as LUVOIR will be necessary to identify multiple biosignatures and establish habitability for Earth-mass exoplanets in the habitable zones of nearby stars.
\end{abstract}

\section{Introduction}

Since 1995, more than 3000 exoplanets have been discovered, mostly via indirect means, ushering in a completely new field of astronomy. In the last decade, about a dozen planets have been directly imaged, including archetypical systems such as

Beth A. Biller

Institute of Astronomy, University of Edinburgh, Edinburgh, UK e-mail: bb@roe.ac.uk

Mickaël Bonnefoy

University of Grenoble e-mail: mickael.bonnefoyeuniv-grenoble-alpes.fr 
HR 8799bcde and $\beta$ Pic $\mathrm{b}$ (Marois et al 2008, 2010; Lagrange et al 2010). Progress in discovering exoplanets has been dramatic, even yielding planets with masses similar to or somewhat larger than the Earth in the habitable zone of their parent star (c.f. Jenkins et al 2015; Anglada-Escudé et al 2016; Kane et al 2016), i.e. at distances from their parent star that may allow liquid water on their surfaces. However, whether or not these planets are capable of hosting life remains an open question the habitable zone planets known to date have only been detected indirectly, meaning we cannot probe their atmospheric composition. Atmospheric characterization of exoplanets via spectroscopy and photometry is necessary to determine if so-called habitable zone planets are truly habitable. Only through studying the atmospheres of exoplanets across a wide parameter space can we truly understand the underlying physical properties of these atmospheres. There are only two methods - direct imaging and transit spectroscopy - that yield photons from the planet itself, enabling direct measurement of atmospheric properties. In this review, we will discuss the current state of the art regarding direct imaging characterization of giant exoplanet atmospheres as well as prospects for future studies of lower mass and even habitable exoplanets with direct imaging.

Such spectroscopic techniques are already in use today to characterize the atmospheres of highly irradiated transiting extrasolar giant planets, mini-Neptunes, and super-Earths (Sing et al 2016, Kreidberg et al 2014a), as well as wide, young giant planets (Macintosh et al 2015; Bonnefoy et al 2016, Chauvin et al 2017). Exoplanet spectroscopy has revealed that clouds (formed of silicate condensates in the case of wide giant exoplanets) are likely common both for transiting and directly imaged planets (Kreidberg et al 2014a; Skemer et al 2012, 2014). While transit spectroscopy is a highly valuable technique for studying exoplanet atmospheres, due to viewing geometry, only $\sim 10 \%$ of planets will have observable transits, and even less for habitable zone planets, which are on average further from their parent star. Thus direct imaging will provide a more complete census of the atmospheres of planets, particularly those around higher mass stars where transits of habitable zone planets are too infrequent to characterize systems on reasonable timescales. Direct imaging spectroscopy of planets also literally provides a different angle compared to the currently dominant transmission spectrum method of studying transiting exoplanet atmospheres. Transmission spectra probe the upper layers of atmospheres at glancing incidence, whereas direct imaging probes straight into these atmospheres, reaching deeper and enabling higher $\mathrm{S} / \mathrm{N}$ detection of interesting spectral features such as biosignatures (Morley et al 2015).

The current cohort of directly imaged planets generally have estimated masses $>2 \mathrm{M}_{\text {Jup }}$ and orbit young stars $(<300 \mathrm{Myr})$. These planets have been imaged primarily in the near- $(1-3 \mu \mathrm{m})$ and mid-IR (3-5 $\mu \mathrm{m})$, near the blackbody peak of their own thermal emission. At these young ages, the planets are still quite warm (effective temperatures, henceforth $T_{\text {eff }}$, between 400 and $2000 \mathrm{~K}$ ) and are hence significantly more self-luminous than at field ages. For such young planets observed at infrared wavelengths, a star-planet contrast ratio in the IR of $\sim 10^{4}-10^{7}$ is expected (Beuzit et al 2008; Macintosh et al 2014). Around nearby, young stars (distances $<100 \mathrm{pc}$ ), these planets are found at projected separations of $0.05-1$ ", although very 
wide exoplanet companions have also been imaged, up to separations of $\sim 2000$ $\mathrm{AU}$ (Naud et al 2014). Such contrasts and resolutions are well within the reach of the current generation of extreme adaptive optics coronagraphs such as SPHERE at VLT (Beuzit et al 2008) and GPI at Gemini (Macintosh et al 2014).

Indeed, the first few directly imaged exoplanets - specifically, HR 8799bcde, $\beta$ Pic b, HD 95086b, and GJ 504b (Marois et al 2008, 2010; Lagrange et al 2010, Rameau et al 2013; Kuzuhara et al 2013) - were actually discovered with facility AO systems without coronagraphs. The advent of extreme adaptive optics coronagraphs with integral field spectrographs has enabled the characterization of spectra and atmospheres for these early discoveries as well as the discovery of additional exoplanet companions (Macintosh et al 2015, Chauvin et al 2017). The initial results from this characterization have been surprising - young exoplanet companions possess significantly redder colors than old field brown dwarfs with similar effective temperatures, suggesting that the much lower surface gravity of the young objects plays a role in atmospheric properties. Additionally, a cohort of young, freefloating planetary mass objects with ages, effective temperatures, estimated masses, and properties similar to the cohort of companion objects has been identified (Gagné et al 2014). This allows us to characterize a much larger sample of objects with comparable properties.

In the next decades, we will characterize this cohort of massive planets in detail, especially at previously inaccessible wavelengths, thanks to the advent of JWST. Space missions such as WFIRST-AFTA will enable the direct detection of Neptune and lower mass objects around Sun-like stars. Extremely large telescopes may image habitable zone exoplanets around nearby $\mathrm{M}$ stars. Finally, in the longer term future, LUVOIR may be able to both detect and characterize Earth-like habitable zone exoplanets around solar-type stars.

In this article, we will summarize the current state of the art in terms of our understanding of young giant planet atmospheres via direct imaging. We will also discuss prospects for deeper characterization of known exoplanets as well as direct imaging of lower mass, closer-in exoplanets in the short term with JWST and in the longer term with the extremely large telescopes, WFIRST-AFTA, HabEx, and LUVOIR. Finally, we will discuss prospects for detecting and characterizing habitable zone, Earthlike planets both from the ground and space.

\section{Methods}

Direct imaging of exoplanets is technically very challenging - imaging even the brightest, young exoplanet companions requires contrasts $>10^{5}$ and resolution into the inner arcsec around the star. Such resolution is possible either from space (where one will be by definition diffraction-limited) or with adaptive optics from the ground. However, even in the diffraction-limited case or after adaptive optics correction, significant quasi-static speckles remain in the inner arcsec, producing a limiting noise floor above the contrasts necessary to image exoplanets (Racine et al 
1999). Thus, various speckle mitigation techniques have been developed to overcome this speckle noise floor.

Numerous speckle-suppression techniques have been proposed and implemented; what these techniques share in common is that they use instrumental methods to distinguish speckles from faint companions. The technique that has become the standard for the field is known as azimuthal differential imaging (henceforth ADI) and uses sky rotation to decorrelate real objects from speckles (Marois et al 2006). Instead of using the telescope rotator to place north up and east left in all images, in an ADI sequence, the rotator is turned off and the field is allowed to rotate with parallactic angle. The speckles, which are instrumental, will continue to appear in roughly the same positions in the image; a real companion or other object in the field, however, will be modulated by the change in parallactic angle. Multiple algorithms have been developed (c.f. LOCI, KLIP, PCA, Andromeda, Lafrenière et al 2007; Soummer et al 2012; Amara and Quanz 2012, Cantalloube et al 2015) to build a model of the speckle pattern in the image on a frame by frame basis, subtract it from each frame, and then stack to produce a "de-speckled" image.

If the adaptive optics correction is high enough (e.g. Strehl ratio $>0.9$, where Strehl ratio=1 would be a perfect diffraction limited image), a coronagraph will also provide significant gains in achievable contrast (Kuchner and Traub 2002). Today, the standard in the field is coronagraphic imaging in tandem with ADI observations. To date, most of the work in this field has been ground-based, as that is where coronagraphs with tight inner working angles are available - HST, while diffraction limited, does not have appropriate coronagraphs for imaging close-in planets. In addition, various ground-based spectroscopic options have become available in the last few years. SPHERE at VLT, GPI at Gemini and now SCExAO+Charis at Subaru (Groff et al 2016) all combine high-contrast coronagraphs with integral field spectrographs to enable low resolution spectroscopy $(R \sim 30-83)$ of exoplanet companions. Integral field spectrographs use image slicers to obtain simultaneous high contrast images in multiple wavelengths, in other words, low resolution spectra. For brighter companions, long-slit spectroscopy can enable higher resolution spectroscopy in combination with high-contrast imaging - e.g. the long-slit spectroscopy modes available with SPHERE at VLT (Vigan et al 2008; Maire et al 2016). In a few cases, it is possible to acquire extremely high contrast spectroscopy in tandem with adaptive optics imaging - this has enabled the first measurement of a rotation period for an extrasolar planet (Snellen et al 2014).

\section{Current results}

Since direct imaging yields luminosities of planets but does not immediately provide a measurement of mass (as the combination of radial velocity and transit detection of the same planet does), objects are studied and classified according to their measured effective temperature $T_{\text {eff }}$. Old field brown dwarfs, young free-floating planetary mass objects, and exoplanet companions to stars are described using the same 
set of spectral types. Unlike stars which remain at the same effective temperature for millions or billions of years due to nuclear core fusion, brown dwarfs and planetary mass objects alike cool monotonically with age, beginning life as hot M-type objects, cooling to the $\mathrm{L}$ spectral type (characterized by very red near-IR colors and silicate condensate clouds (Kirkpatrick|2005), the T spectral type (characterized by blue near-IR colors and strong methane absorption at $1.6 \mu \mathrm{m}$ and $2.2 \mu \mathrm{m}$, Kirkpatrick 2005) and eventually to the very cool Y spectral type (Cushing et al 2011; Kirkpatrick et al 2012, see Fig. 11). Thus, there is an age / mass / temperature degeneracy for these objects. Additionally, it has become clear that low surface gravity in younger and lower mass objects can significantly affect the spectra of these objects and also the $\mathrm{T}_{\text {eff }}$ at which these objects transition between spectral types (Barman et al 2011).

The number of directly imaged exoplanets remains small, both because of the technical difficulty of their detection, and also because initial surveys have demonstrated that giant exoplanets are rare at separations $>30 \mathrm{AU}$, where we can readily detect them (c.f. Biller et al 2013b; Brandt et al 2014). However, a larger, statistically significant number of objects with similar properties are available to study if we consider as well free-floating planetary mass objects with similar ages. Many direct-imaging planet host stars known to date (e.g. HR 8799, $\beta$ Pic) are members of nearby young moving groups - aggregations of young stars (10-300 Myr) in the solar neighborhood that were formed in the same star-forming cloud and still share similar sky motions, even though the cloud materials have long since dissipated (Zuckerman and Song 2004, Schlieder et al 2012, also, for an example of a recent membership search of such a group). In the last few years, searches for new moving group members using advanced Bayesian techniques have begun to identify planetary mass or very low mass brown dwarf $\left(<25 M_{\text {Jup }}\right)$ members of these groups (see c.f. Gagné et al 2014, 2015, Faherty et al 2016). On order 30 such objects have been identified to date. These objects do not necessarily share the same formation mechanism as young exoplanet companions to stars, but they do share similar ages, masses, and compositions and are unobscured by a nearby bright star. Thus, they serve as important and much more easily studied proxies to directly imaged planets. Similarly to directly imaged planets, young free-floating planetary mass objects possess significantly redder colors than field brown dwarfs with similar spectral types. For the rest of this section, we consider results from free-floating planetary mass objects alongside results from bonafide planetary mass companions.

\section{Initial photometric measurements}

Given the age / temperature / luminosity degeneracy for substellar objects, it was initially expected that directly imaged exoplanets should have similar colors and spectral types as T spectral type brown dwarfs, as both directly imaged exoplanets and more massive but older $\mathrm{T}$ type brown dwarfs should have expected $T_{\text {eff }}<1400 \mathrm{~K}$ and similar compositions. T-type brown dwarfs possess notable methane absorp- 


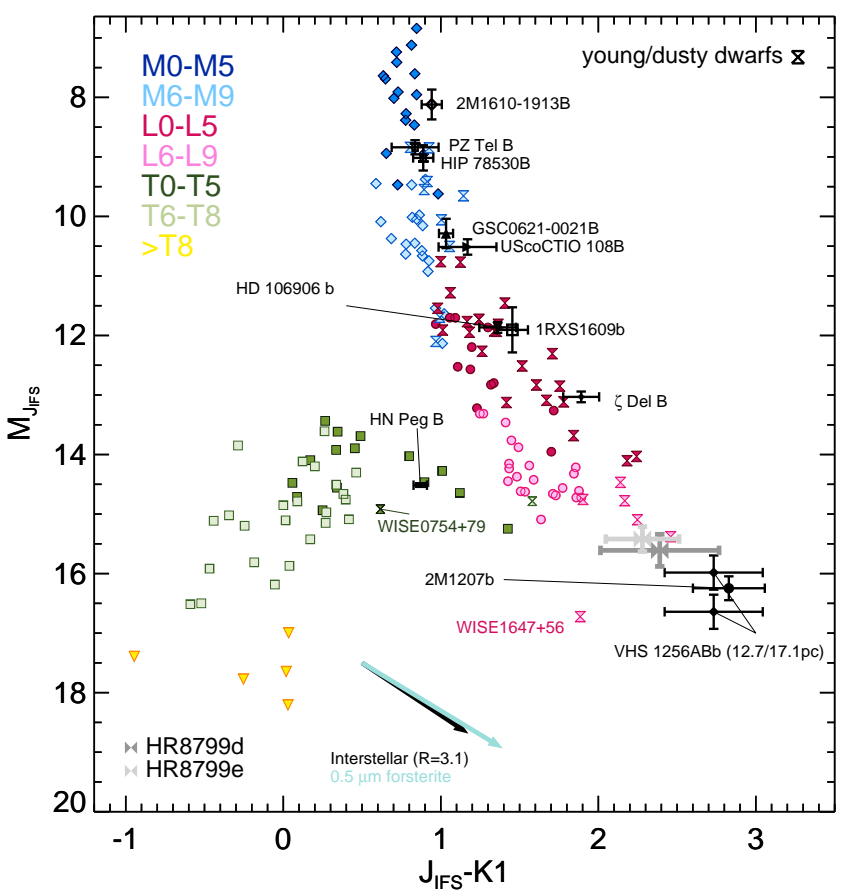

Fig. 1 Color-color diagrams for HR 8799bcde, free-floating planetary mass objects and wide planetary mass companions. The $J_{I F S}$ magnitudes correspond to a virtual filter encompasing the 1.20$1.32 \mu \mathrm{m}$ range of the integral field spectrograph (IFS) of SPHERE. The K1 filter has a 2.06-2.16 $\mu \mathrm{m}$ passband. Field dwarfs are overplotted for comparison. All exoplanets and brown dwarfs begin life as M-type objects, cooling and moving red-wards to the L spectral type, then transitioning to the comparatively blue $\mathrm{T}$ spectral type (due to strong methane absorption that removes flux predominantly in the $\mathrm{K}$ band). Compared to field brown dwarfs, young planetary mass objects retain red colors and $\mathrm{L}$ spectral types down to much lower $T_{\mathrm{eff}}$.

tion features at 1.6 and $2.2 \mu \mathrm{m}$; early searches for exoplanet companions specifically targeted deep methane absorption expected in these objects (Biller et al 2007). However, in contrast to expectation, the first planetary mass companions imaged had extremely red colors relative to field dwarfs with similar $T_{\text {eff }}$ and no methane absorption. In Fig. 1. we plot $J-K$ color-magnitude diagram and report the photometry of young companions, including the those of the planets HR8799 d and $\mathrm{e}$, as well as a subset of other young free-floating and companion planetary mass objects. M, L, and T field brown dwarfs are plotted as well. The young planetary mass objects plotted clearly have considerably redder $J-H 2$ colors than their field counterparts with similar $T_{\text {eff }}$. These objects, however, also must have lower surface gravities compared to the field dwarfs, which should affect atmospheric structure. Various groups have suggested that thick and/or patchy clouds are necessary to ex- 
plain the extremely red photometry measured for these objects (Barman et al|2011, Madhusudhan et al (2011).

\section{High contrast spectroscopy}

With the advent of high-contrast imagers with integral field spectrographs such as SPHERE and GPI, low resolution (R 30-83) near-IR spectra have been acquired for several bright planets (Bonnefoy et al 2016; Zurlo et al 2016; Bonnefoy et al 2014b, Ingraham et al 2014, Chilcote et al 2015, De Rosa et al 2016; Macintosh et al 2015; Samland et al 2017), allowing for a more detailed inspection of atmospheric properties. We summarize these results, starting with the highest effective temperature planets and continuing on with decreasing temperature.

$\beta$ Pic $\mathrm{b}$ is a $\sim 8-13 M_{\text {Jup }}$ companion $8-10$ AU from the $\sim 20$ Myr intermediate mass star $\beta$ Pic. Given the higher mass of its primary relative to the Sun, it is very much like a higher mass analogue to Saturn in our own solar system and could have formed in a similar way as Jupiter. In 2014, the Gemini Planet Imager instrument (GPI) was used to capture the first low-resolution near-infrared $(1.1-1.8 \mu \mathrm{m})$ spectra of the exoplanet (Bonnefoy et al 2014b; Chilcote et al 2015). The spectrum displays water band absorptions and is characteristic of young low-gravity objects. This spectrum and additional photometry from 2 to $5 \mu \mathrm{m}$ shows that the planet has an early L spectral type, an effective temperature around $1700 \mathrm{~K}$ (Bonnefoy et al 2014b, Morzinski et al 2015), and a radius of $\sim 1.5 R_{\text {Jup }}$.

The four planets orbiting the intermediate mass star HR 8799 all have L/T spectral types and estimated masses between 5 and $7 M_{\text {Jup }}$. They offer the opportunity to perform comparative planetology beyond our Solar System. In the past few years, images of that system have been gathered from 0.9 to $5 \mu \mathrm{m}$. Using spectrophotometry at mid-IR wavelengths from 3-5 $\mu \mathrm{m}$, Skemer et al (2012) and Skemer et al (2014) find that patchy cloud models and/or non-equilibrium chemistry are necessary to model the spectral structure at these wavelengths for HR $8799 \mathrm{~b}$, c, and d. More recently, the instruments P1640, SPHERE, and GPI yielded low-resolution near-infrared (0.9-2.5 $\mu \mathrm{m}$ ) spectra of each planets (Oppenheimer et al |2013, Ingraham et al 2014:Zurlo et al 2016) which can be compared to those of known objects (see Fig. 2). The spectra of the two innermost planets are similar to those of young and peculiar late-L brown dwarfs identified in the solar neighborhood (Bonnefoy et al 2016). This confirms that the peculiar properties of the planet emergent fluxes are related to their low surface gravity. The spectra of the two outermost planets cannot be represented by those of any other known object. But free-floating analogues of HR 8799b and c may exist in the Solar neighborhood or in young clusters and remain to be found.

The increased sensitivity of the new generation of direct imaging instrument SPHERE and GPI enable detection of fainter and cooler objects closer to their parent stars. 51 Eri b (Macintosh et al 2015) is the first exoplanet to be identified by these instruments and is also the first planet to clearly exhibit methane absorption 


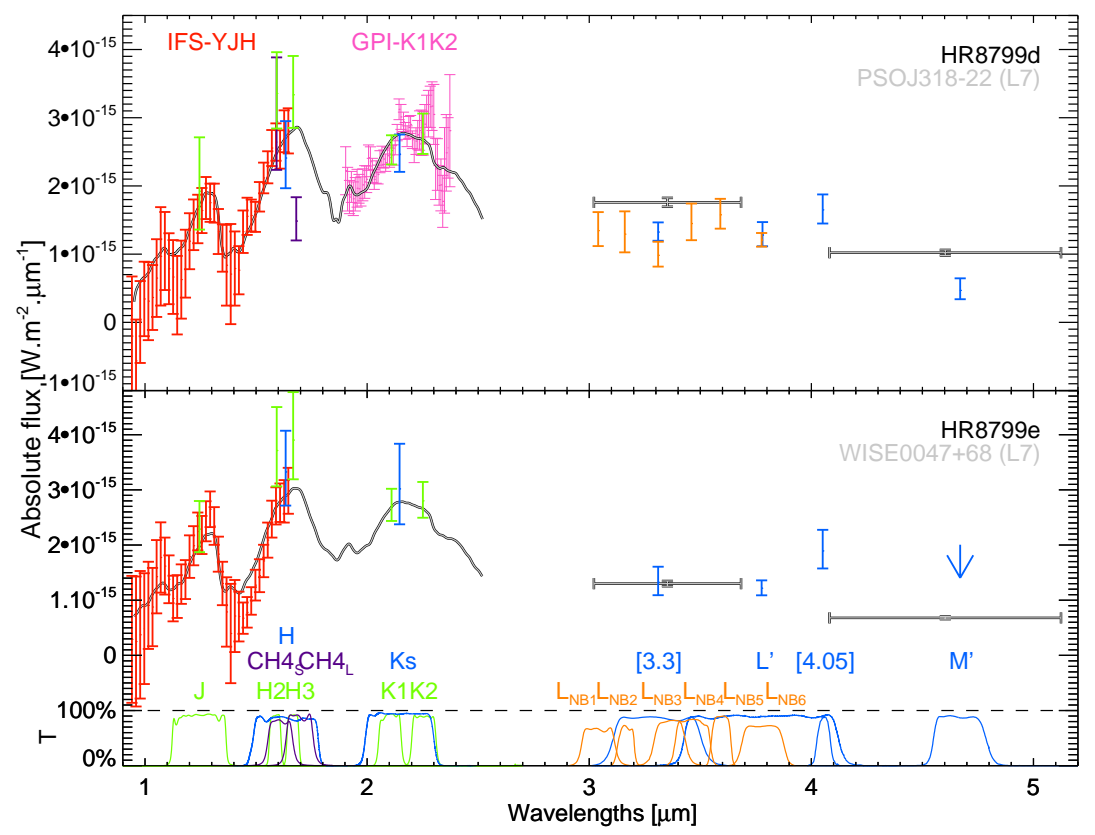

Fig. 2 Near-IR spectra and mid-IR spectrophotometry for the planets HR 8799d and e. with data from Oppenheimer et al (2013); Skemer et al 2012, 2014); Ingraham et al 2014); Bonnefoy et al (2016); Zurlo et al (2016).

features, shown in Fig. 3. Only two other young wide-orbit companions show similar absorption features (GU Psc b and HN Peg b; Naud et al 2014, Luhman et al 2007), but is likely that those objects did not form like planets. 51 Eri b is quite a bit cooler than other imaged planets and suggests that the L/T transition occurs at considerably lower $T_{\text {eff }}$ for low surface gravity planets than for high surface gravity brown dwarfs.

\section{Variability}

Most direct imaging observations of exoplanets to date have had short durations of 2 hours or less, and thus probe planet properties in a time-averaged and spatially unresolved manner. However, new exoplanet imagers such as GPI and SPHERE offer the possibility to directly image exoplanets with cadences of 20-30 minutes, allowing us to search for rotationally modulated variability. Detectable rotationally modulated variability would be produced in giant exoplanets in the presence of: 1) relatively short rotation periods (i.e. observable on the timescales of a night or two) and 2) surface inhomogeneities, potentially caused by inhomogeneous cloud cover 


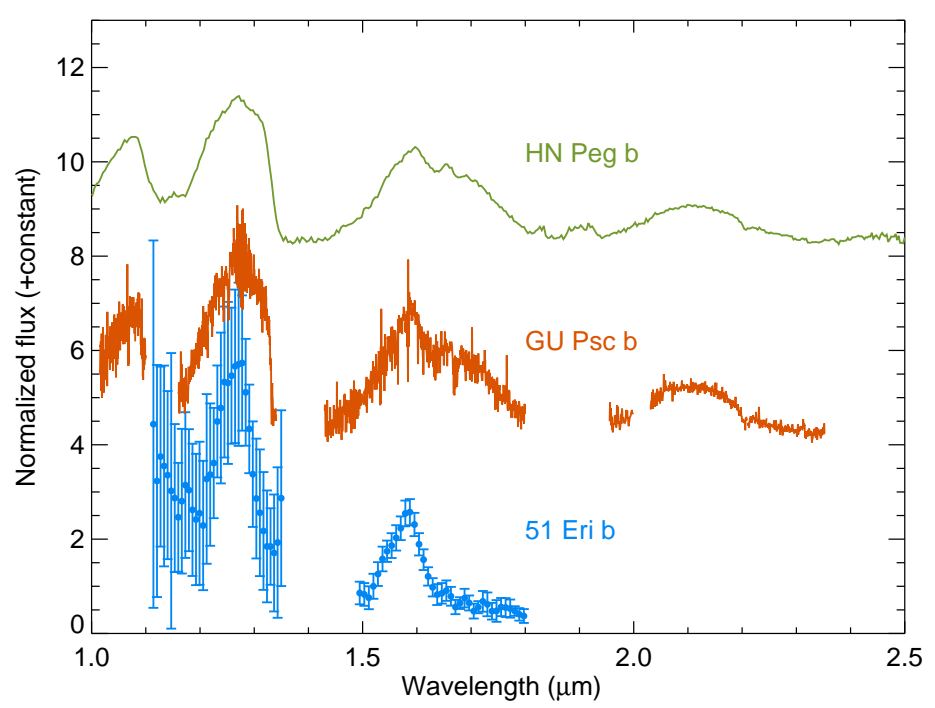

Fig. 3 Spectra of young companions 51 Eri b, HN Peg b, and GU Psc b taken from Macintosh et al (2015); Naud et al (2014); Luhman et al (2007) with methane absorption at $1.6 \mu \mathrm{m}$ and $2.2 \mu \mathrm{m}$. The young Jupiter analogue 51 Eri b is the first clearly T-type planet.

Apai et al (2013) or thermo-chemical instabilities Tremblin et al (2015, 2016). Both of these conditions are fulfilled for brown dwarfs and appear also to be fulfilled for young giant planets.

Brown dwarfs are known fast rotators, with periods of 3-20 hours (Zapatero Osorio et al 2006). Preliminarily, one might expect young giant exoplanets to be somewhat slower rotators, as their younger ages imply that they have not had as much chance to spin up as they contract. However, initial measurements of rotational period for planetary mass objects (both companions and free-floating) have found periods generally less than 20 hours (in 4 out of 5 cases to date). The first such measurement, of the giant exoplanet $\beta$ Pic $\mathrm{b}$, derived a rotation period of 7-9 hours for this planet from the Doppler broadening of spectral features in the planet's atmosphere (Snellen et al 2014). From its photometric variability, 2MASSW J1207334-393254b (henceforth 2M1207b), a young planetary mass companion to a 20-30 $M_{J u p}$ brown dwarf, was found to have a rotation period of $\sim 11$ hours (Zhou et al 2016). The free-floating planetary mass object PSO J318.5-22 has a rotation period between 5 and 10 hours (Biller et al 2015; Allers et al 2016) and the very low mass brown dwarf W0047 $\left(<20 M_{\text {Jup }}\right)$ has a rotation period of $\sim 13$ hours (Lew et al 2016). Of these early measurements, the one outlier is the rather more massive companion GQ Lup B $\left(\sim 40 M_{\text {Jup }}\right)$, which is a much slower rotator with a period of 82 days in the case that the orientation of the spin axis is edge-on (Schwarz et al 2016). However, taken as a whole, it is not implausible that exoplanet companions other than $\beta$ Pic $b$ may have relatively fast rotation periods. 
Surface inhomogeneities provides the second ingredient for quasi-periodic photometric variability. Such variability is already commonly observed for field brown dwarfs (Radigan et al 2014; Wilson et al 2014; Radigan 2014; Metchev et al 2015) and is often attributed to patchy thin and thick clouds, especially along the L/T transition (Apai et al 2013), although recently thermochemical instabilities have also been proposed as a source of surface inhomogeneities (Tremblin et al 2015, 2016). Young exoplanets should possess similar clouds (generally composed of hot silicate particles; Barman et al 2011; Skemer et al 2012, 2014), although the structure of these clouds may vary due to the lower surface gravities of these objects relative to field brown dwarfs. At least preliminarily, it seems likely that young exoplanets should also be similarly variable.

Only two known systems have planets sufficiently bright for variability monitoring on reasonable cadences with current instruments, specifically HR $8799 \mathrm{bcde}$ and $\beta$ Pic b. One search for variability in exoplanet companions to main sequence stars has been published to date - Apai et al (2016) obtained SPHERE science verification data to monitor for variability in HR 8799bcd over several nights in December 2015. As HR 8799 is only available at reasonable airmasses at the very beginning of the night in December, each observation was only 30 minutes long. Thus, while Apai et al (2016) demonstrated that satellite spot-modulated artificial planet-injection based photometry (SMAP) produced a significant $(\sim 3)$ gain in photometric accuracy over standard aperture-based photometry, they did not possess the cadence to detect variability on time scales similar to the expected rotation periods of the planets.

While the extreme photometric stability expected with JWST will open up a few more systems for variability monitoring, free-floating planetary mass objects and young, very low mass brown dwarfs provide another (considerably larger) sample of similar objects for variability studies. Preliminarily, these objects seem to be as variable or more variable than field brown dwarfs. Biller et al (2015) published the first detection of such variability for the $\sim 8 M_{J u p}$ free-floating planetary mass object PSO J318.5-22 Liu et al (2013), with an amplitude of 7-10\% in J. Soon thereafter, Lew et al (2016) reported high-amplitude variability in the young, very low mass brown dwarf WISEP J004701.06+680352.1 (henceforth W0047, a member of the AB Dor moving group). Both of these objects have late L spectral types and provide a striking contrast to variability detected to date for field brown dwarfs. Among field brown dwarfs, $\mathrm{L} / \mathrm{T}$ transition brown dwarfs display the highest variability amplitudes (Radigan et al 2014), interpreted as break-up of clouds along the $\mathrm{L}$ to $\mathrm{T}$ spectral type transition producing patchiness and hence variability. In contrast PSO J318.5-22 and W0047 have late L spectral types, and presumably thick dusty clouds - but variability amplitudes similar to L/T transition brown dwarfs (and are in fact the highest amplitude variables among the entire L spectral type), suggesting distinctly different atmospheric structure compared to higher-gravity field objects. Lower level variability with a period of 11 hours has also been detected for $2 \mathrm{M} 1207 \mathrm{~b}$, a late-L planetary mass companion to a young brown dwarf (Yang et al 2016). Lightcurves for variable exoplanet analogues are shown in Fig.4 

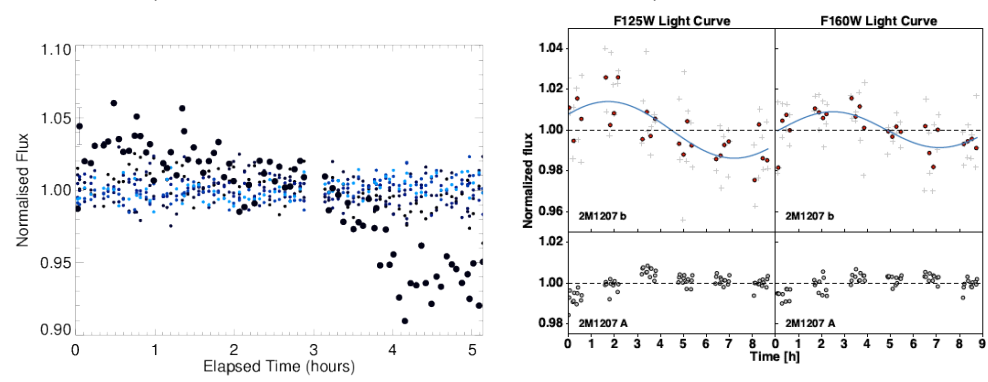

W0047, Lew et al. 2016

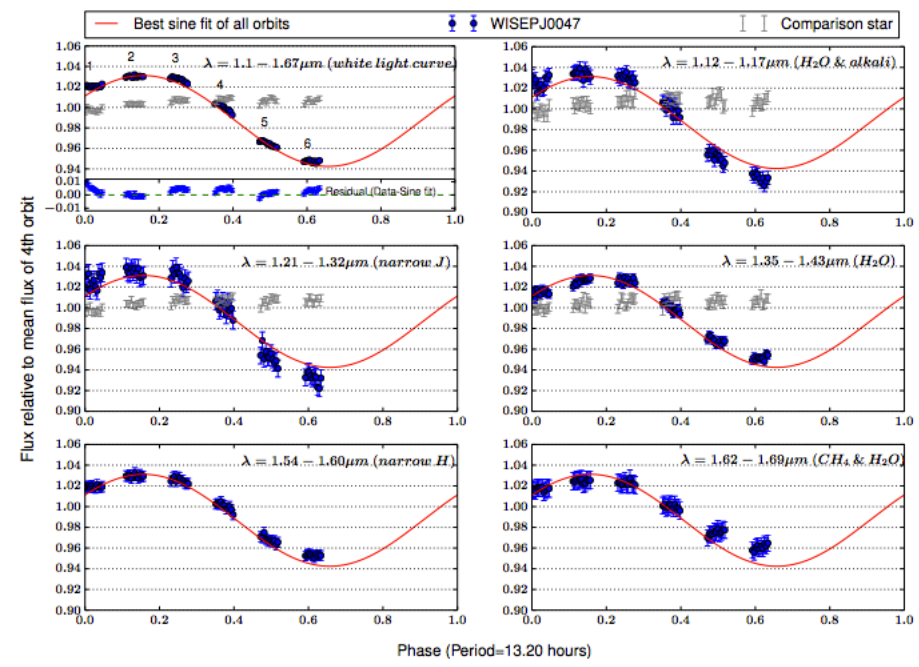

Fig. 4 Variability of young free-floating planetary mass objects and wide companions, figures from Biller et al (2015); Zhou et al (2016); Lew et al (2016). Preliminarily, quasi-periodic variability potentially driven by cloud features appears to be as common or more common in low surface gravity planetary mass objects compared to higher surface gravity field brown dwarfs.

Variability eventually enables mapping of planetary surfaces, as the time-dimension allows us to probe different parts of the surface at different times in the observation. For brown dwarfs, significant effort has already gone in to reconstructing surface features based on spectroscopic variability monitoring (Apai et al 2013; Karalidi et al 2015, 2016). Indeed, for the closest brown dwarf to the Earth, the L/T transition binary Luhman 16AB (Luhman 2013), the surface of the highly variable B component (Gillon et al 2013, Biller et al 2013a) has been mapped via the Doppler imaging technique (Crossfield et al 2014), presented here in Fig. 5. While similar studies for exoplanets are still in their infancy, eventually similar maps will be produced for exoplanets as well - in particular, the high-resolution spectrograph 
planned for the METIS instrument on the E-ELT may provide Doppler images of the surface of $\beta$ Pic $b$, a fast rotator with a 7-9 hour period.

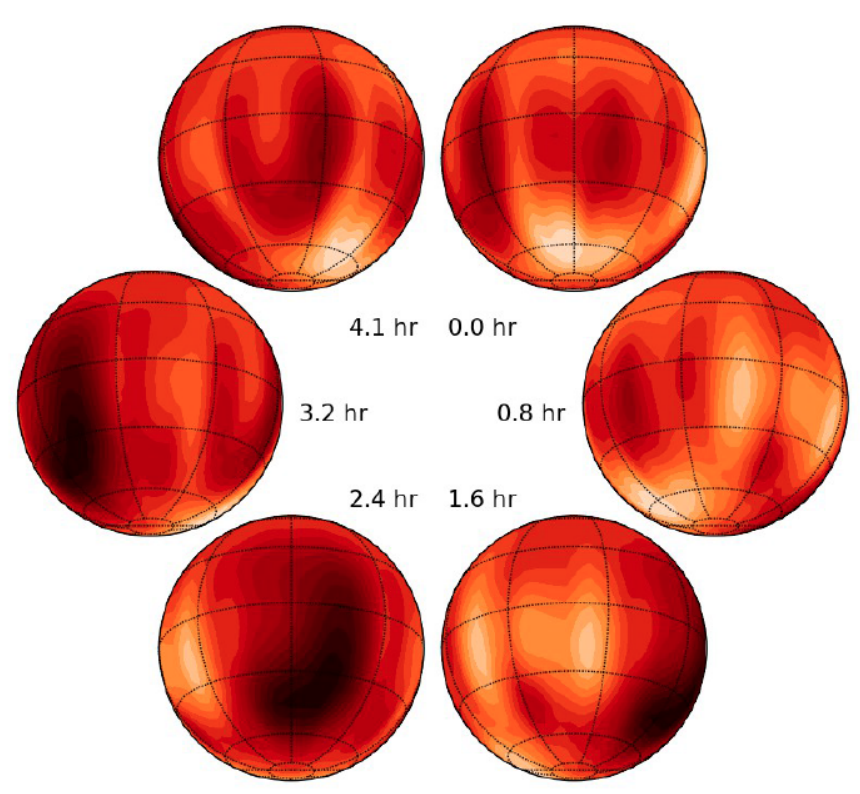

Fig. 5 Doppler map of the early T variable brown dwarf Luhman 16B, from Crossfield et al (2014). High-resolution spectrographs on extremely large telescopes will enable similar mapping of directly imaged exoplanet companions.

\section{Very young accreting planets}

Young companions are thought to be surrounded by circumplanetary disks, from which they acquire their mass (e.g. Quillen and Trilling 1998). The accretion of the hydrogen gas contained in these disks produces characteristic emission lines in the companion spectra in the optical $\left(H_{\alpha}, 656 \mathrm{~nm}\right)$ and in the near-infrared $\left(\mathrm{Pa}_{\beta}, 1.282\right.$ $\mu \mathrm{m})$. Up to now, those signatures have been found for five young (1-11 Myr) companions with masses between 11 and $17 \mathrm{M}_{\text {Jup }}$ and large projected separations of several hundreds of au (Seifahrt et al 2007: Schmidt et al 2008; Bowler et al 2011, 2014, Bonnefoy et al 2014a: Zhou et al 2014). The line intensities were used to estimate gas accretion rate (e.g. Zhou et al 2014) ranging from $10^{-11.5}$ to $10^{-9.3} M_{\odot} /$ year. Similar accretion rates are found for free-floating brown dwarfs down to $11 M_{J u p}$ (Natta et al 2004, Joergens et al 2013).

The intensity of those lines can evolve in time (Fig. 6). In 2005, the companion GQ Lup b displayed a strong Paschen $\beta$ line which had nearly vanished in 
2007 (Seifahrt et al 2007); Lavigne et al 2009). The same phenomenon was observed more recently for the companion GSC 06214-00210 b (Lachapelle et al 2015) in the near-infrared and in the optical for GQ Lup b (Wu et al 2017). The amplitude and timescales of the line variability could provide precious clues on the way the planets accrete their material, and how their physical properties (temperature, radius, luminosity) evolve in time (up to $\sim 100 \mathrm{Myr}$; Marley et al 2007, Baraffe et al 2009).

Recent observations with ALMA have set constraints on the mass reservoir surrounding those objects (Caceres et al 2015; Bowler et al 2015, Kraus et al 2015, MacGregor et al 2017, Wu et al 2017). The accretion rate and disk mass are related to the circumplanetary disk lifetime and indirectly determine the formation mode and timescale of the gas giant planets.

The line emission can be exploited to detect young accreting planets within circumstellar disks. In the optical, the continuum of the planet is faint so that carefully designed narrow-band filters centered on an off the $H_{\alpha}$ line can capture the emission of the planet while removing the stellar flux. This technique has been implemented on the new generation of extreme adaptive optics instruments such as MagAO on the Magellan telescope (Close et al 2013) and SPHERE at the VLT (Beuzit et al 2008), and has already yielded the detection of an accreting stellar companion to the Herbig star HD142527 (Close et al|2014) and of a Jovian planet around the star LkCa 15 (Sallum et al 2015).

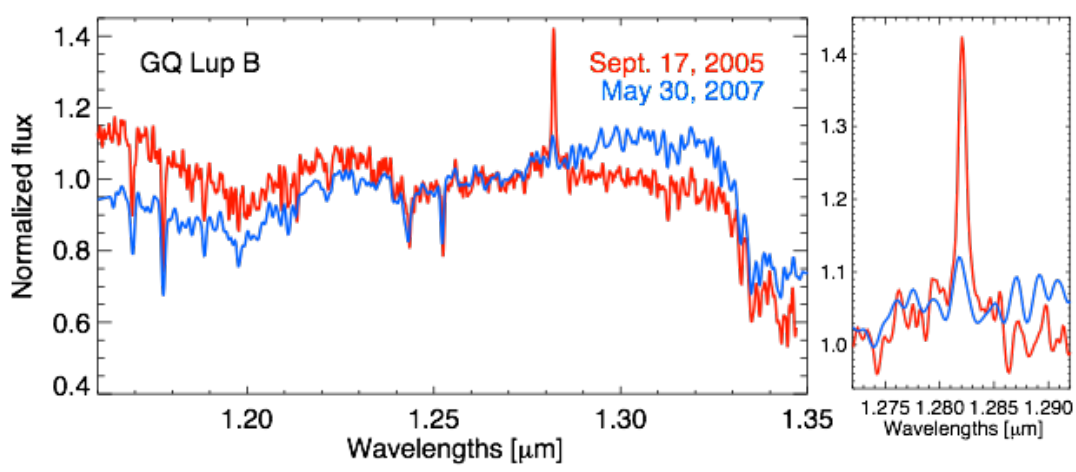

Fig. 6 Near-infrared spectra of the low-mass companion GQ Lup B. In 2005, the companion spectrum exhibited a strong Paschen $\beta$ emission line at $1.282 \mu \mathrm{m}$ (Seifahrt et al 2007). This line had nearly vanished in a spectrum taken in 2007 (Lavigne et al 2009). 


\section{Prospects for the future}

We have summarized the current state-of-the-art regarding the characterization of exoplanet atmospheres via direct imaging with current (largely ground-based) instruments. This field is pushed forward by technology development and the advent of new telescopes and instruments. A few critical developments will produce notable steps forward in this field. In the next 2 years, the advent of JWST will lead to much more detailed characterization of bright, relatively wide exoplanet companions and free-floating planetary mass objects. In 10 years, the extremely large telescopes will enable in-depth characterization of Neptune to Jupiter-mass exoplanets and the potential to directly detect super-Earths around very nearby M-stars. WFIRST-AFTA will directly image super-Earths to Neptune planets and in the very long term future, HabEx and LUVOIR may image the first habitable exoplanets around Sun-like stars.

\section{The next 5-10 years}

To date, no directly imaged exoplanet has been characterized at wavelengths longer than $5 \mu \mathrm{m}$. This is because current facilities are ground based and thus suffer from the very high sky background at these long wavelengths (since the blackbody peak for the Earth's atmosphere is at around $10 \mu \mathrm{m}$ so literally everything around your telescope is glowing). To effectively observe at these wavelengths, it is imperative to work from space, above the Earth's atmosphere. The mid-IR Spitzer space telescope was capable of this during the cooled Spitzer mission, but the coarse resolution of Spitzer and lack of coronagraphs precluded studying close companion objects. The MIRI instrument in JWST possesses four-quadrant phase masks at 10.65, 11.4 and $15.5 \mu \mathrm{m}$, and a Lyot coronograph at $23 \mu \mathrm{m}$, allowing the study of exoplanet companions at these wavelengths for the first time (Boccaletti et al 2015). The fourquadrant phase masks have inner working angles at approximately $\lambda / D$, limiting studies to companions at separations greater than $0.35-0.5 "$. Nonetheless, this will enable long wavelength photometry of much of the cohort of directly-imaged exoplanet companions.

The high quality spectra and photometry of giant exoplanets obtained with the JWST will be highly suitable to atmospheric retrieval studies. The retrieval method uses a Bayesian framework (Markov-chain Monte-Carlo, nested sampling) to represent a set of data and associated errors and infer posterior distributions on each free parameter considered in the models. The method has been successfully applied to the spectra of brown dwarf companions (Line et al 2014, 2015; Burningham et al 2017) and of transiting exoplanets (e.g. Kreidberg et al 2014b, Benneke 2015). It can set constraints on their atmospheric structures and composition. In the JWST era, the method promises to provide unprecedented clues to the origin of giant planets (Lee et al 2013; Lavie et al 2016) through the quantification of the abundances of carbon $(\mathrm{C})$, oxygen $(\mathrm{O})$, and hydrogen $(\mathrm{H})$ provided that the formation of the 
cloud deck in the atmosphere of those planets is properly modelled (Helling et al 2014; Burningham et al 2017). The $\mathrm{C} / \mathrm{H}$ and $\mathrm{C} / \mathrm{O}$ ratio should be good tracers of the formation mode of the planets and of their formation zone within the disks (e.g. Helled and Schubert 2009, Öberg et al 2011, Öberg and Bergin 2016). Comparing the formation zone to the present-day location of the planets could give clues on the efficiency of the migration processes and on past dynamical interactions, vital information needed to constrain and enrich planet formation theory (Mordasini et al 2009).

The coupling of high contrast imaging and high-resolution spectroscopy offers additional prospects for detailed studies of the atmospheric physics of giant planets. Once the high resolution near-infrared spectrograph CRIRES at the VLT is back on sky in 2018 (in the new CRIRES+ mode with increased sensitivity and wavelength coverage, Dorn et al 2016), it will be used as the backend for the SPHERE planet-imager, providing very high resolution star+exoplanet spectra. This should enable the measurement of the rotation velocity of additional planets (Snellen et al 2014, 2015) and will also provide an additional opportunity to apply retrieval methods to derive more accurate information on atmospheric states and chemical abundances. The high-resolution near-infrared spectrographs CARMENES (Quirrenbach et al 2016) and SPIRou (Artigau et al 2014) will also provide spectra of tens of free-floating exoplanet analogues (young moving group members discussed previously). The high quality high resolution spectra of those objects will serve as proxies for understanding exoplanet physics. This may also allow us to determine if those objects were formerly companions that were ejected from nascent planetary systems due to dynamical interactions with other planets.

\section{Prospects for detecting and characterizing habitable planets with direct imaging}

In the next few years, JWST will obtain transit spectroscopy for a handful of nearby M-star habitable zone planets potentially to be discovered by the TESS mission (Ricker et al 2014; Sullivan et al 2015). However, only a small percentage of habitable zone exoplanets are expected to transit. In the next 10-20 years, direct imaging will eventually enable characterization of a larger sample of habitable zone exoplanets.

\section{WISE 0855 - the coolest atmosphere imaged to date}

While not likely habitable itself, the extremely cool, nearby brown dwarf WISE 0855 (Luhman 2014) has $T_{\text {eff }} \sim 270 K$, around the freezing point of water. It is the coldest object ever imaged outside of our own solar system and gives us a sneak peak of what a habitable zone temperature atmosphere looks like. Skemer et al (2016) obtained the first spectrum of this object, at mid-IR wavelengths from 
4.5 to $5.2 \mu \mathrm{m}$, presented here in Fig. 7. The spectrum of this object shows clear evidence for the presence of water clouds, but does not show the strong $\mathrm{PH}_{3}$ absorption seen in Jupiter's spectrum. Given the presence of water clouds, Yates et al (2016) suggest that very cool brown dwarfs such as WISE 0855 may have an "atmospheric habitable zone" - a range in its atmosphere with liquid water clouds as well as temperatures and pressures appropriate for Earth-centric life.

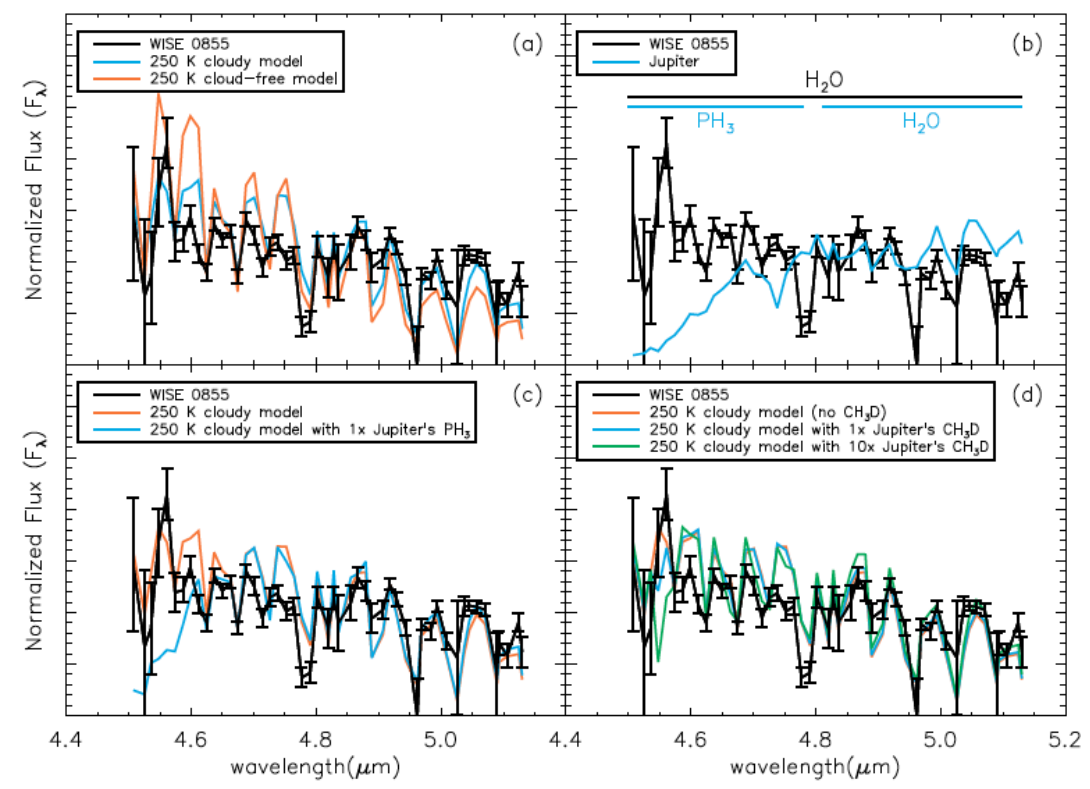

Fig. 7 Spectrum of the extremely cold $\left(T_{\text {eff }} \sim 270 K\right)$ brown dwarf WISE 0855, from Skemer et al (2016). Water clouds are necessary to reproduce the observed spectral features. The phosphine $\left(\mathrm{PH}_{3}\right)$ absorption features seen in Jupiter's spectrum are not apparent in WISE 0855.

\section{Methods for Detecting and Characterizing Habitable Zone Planets}

Other than transit spectroscopy, three other methods will be able to characterize habitable zone exoplanets over the next decades:

1. Thermal Phase Curves - Whether or not a planet transits, a tidally-locked planet may be detectable from its thermal phase curve - the difference in temperature between the day side and night side of the planet, evident in the star-planet spectrum in the mid-IR (Gaidos and Williams 2004, Seager and Deming|2009; Selsis et al 2011, 2013;: Maurin et al 2012;: Turbet et al 2016; Kreidberg and Loeb 2016).

2. High-contrast imaging + high-resolution spectroscopy The combination of high-resolution ground-based optical spectroscopy with high-contrast imaging 
can potentially characterize known planets (Riaud and Schneider 2007; Snellen et al 2015). First, high-contrast imaging is used to achieve $\sim 10^{5}$ contrasts in the inner arcsec, then high-resolution spectroscopy can distinguish between the stellar spectrum vs. the Doppler-shifted planet spectrum. This technique has already been used to measure a rotation period for the young, giant exoplanet $\beta$ Pic b (Snellen et al 2014). Snellen et al (2015) predict that a $R=1.5 R_{\text {earth }}$ planet with a twin-Earth thermal spectrum orbiting $\alpha$ Cen A could be detected in one night of E-ELT time using this method.

3. Direct imaging Finally, in a decade, next-generation ground based extremely large telescopes (ELTs) as well as space coronagraphs such as WFIRST-AFTA will be able to detect very nearby habitable zone planets orbiting M stars (Crossfield 2014) as well as older gas giants around G stars (need citation). In the longer term future, space missions such as LUVOIR will directly image habitable zone planets around G stars.

\section{Proxima Centauri b}

2016 yielded the very exciting discovery of a habitable zone planet around Proxima Centauri (Anglada-Escudé et al 2016), the closest star to the Sun. Proxima Centauri b has a minimum mass of $1.3 M_{\text {Earth }}$ and an orbital period of 11 days, placing it squarely in the middle of the habitable zone around this low mass star. While several groups have already modeled the potential habitability of this planet (Ribas et al 2016, Turbet et al 2016, Barnes et al 2016, Meadows et al 2016; Goldblatt 2016; O'Malley-James and Kaltenegger 2016), as it has only been observed indirectly, whether or not the planet is habitable is an open question. Additionally, there are a number of factors that make habitability less likely - specifically, its somewhat higher mass than the Earth (which is highly dependent on orbital inclination with possible values up to 8 earth masses, Kane et al 2017), the fact that it is likely tidally locked, as well as the much higher radiation environment expected for this planet around an active $\mathrm{M}$ star relative to our own fairly quiet $\mathrm{G}$ star (Turbet et al 2016, Garraffo et al 2016; Atri 2017; Davenport et al 2016). (For an excellent recent review of work to date on potential habitability of M-star planets, see Shields et al (2016).) Thus, direct atmospheric characterization is vital to determine if the planet can have liquid water on its surface or perhaps even displays evidence for biosignatures. The transit probability for Proxima Centauri $b$ is only $1.5 \%$ and initial optical searches have yielded no conclusive evidence of transits (Kipping et al 2017). Kreidberg and Loeb (2016) model the possibility of detecting Proxima Cen b via its thermal light curves with JWST. This is highly dependent on the atmosphere of the planet - Kreidberg and Loeb (2016) predict that they can distinguish between a bare rocky planet and a planet with $35 \%$ heat redistribution to the nightside at the 4- $\sigma$ level, but very long integration times (on order of a few months) would be necessary to detect the $9.8 \mu \mathrm{m}$ ozone band in the case of an Earth-like atmosphere. Thus, direct imaging will likely provide the first opportunity to characterize this planet and determine if it is truly habitable. 
The estimated star-planet contrast for Proxima Centauri $b$ is $\sim 10^{7}$ in the thermal IR. While this seems extreme, this is is still moderate compared to the $10^{10}$ contrast expected for a habitable zone planet observed in reflected light around a G star Trauger and Traub (2007). The current generation of planet-imagers such as GPI and SPHERE already reach such contrasts - but not at the separations necessary to image Proxima Centauri $b$, as Proxima Centauri $b$ is expected to be within 40 mas of A (Turbet et al 2016). Thus, detecting Proxima Centauri b requires currently achievable contrasts, but diffraction-limited performance on a 30-40 m class telescope. Likely in a decade, instruments such as METIS on the E-ELT will be the first to image Proxima Centauri b, if it is reflective enough. However, while ELTs may detect Proxima Centauri b, they may not be suitable for the task of detecting a wide range of biosignatures and demonstrating dis-equilibrium chemistry. As many potential biosignature gas features may also have abiotic sources (e.g. ozone DomagalGoldman et al 2014, Luger and Barnes 2015, Harman et al 2015), coverage of multiple biosignatures and clear evidence of dis-equilibrium chemistry will be necessary to prove habitability. Many such signatures are at UV wavelengths, inaccessible with ground-based ELTs. Luger et al (2016) suggest aurorae on Proxima Centauri $\mathrm{b}$ could also be detected via direct imaging with an extreme AO coronagraph on an ELT.

In the shorter term, the technique of high-contrast imaging + high-resolution spectroscopy may be able to detect Proxima Centauri b, but only with considerable expenditure of observing time. Lovis et al (2016) find 20-40 nights of telescope time with upgraded versions of the high-contrast imager SPHERE and the high-resolution spectrograph ESPRESSO would be required for a $5-\sigma$ detection of Proxima Centauri b, assuming an Earth-like atmosphere. To search for biosignatures (the combination of $O_{2}$, water vapor, and methane) would require 60 nights of telescope time for a $3.6-\sigma$ detection.

Proxima Centauri's higher mass neighbors may also be excellent targets to search for habitable zone planets. Belikov et al (2015) argue that because $\alpha$ Centauri A and $\mathrm{B}$ are so nearby and thus have a much higher apparent habitable zone size compared to any other FGKM stars in the sky, a habitable zone exoplanet around $\alpha$ Centauri A or B could be imaged with a $30-45 \mathrm{~cm}$ space telescope and advanced post-processing techniques.

\section{Other habitable zone exoplanets}

Most of the currently known habitable zone exoplanets (largely Kepler discoveries around $\mathrm{K}$ and $\mathrm{M}$ stars, Kane et al 2016) will remain out of the reach of direct imaging, given their tight separations from their parent stars. However, similar exoplanets around very nearby stars may be imaged with extremely large telescopes. Especially for planets around nearby M-stars, the limiting factor is not contrast, but resolution. One way to probe even closer in to a star is to combine high-contrast imaging with high-resolution spectroscopy (Snellen et al 2015). As mentioned previously, an upgraded SPHERE + ESPRESSO at the VLT may be able to detect 
Proxima Centauri $b$ in this manner (Lovis et al 2016). Another way to increase resolution is simply to use a bigger telescope with adaptive optics correction to ensure diffraction-limited performance. Thus, the advent of 30-40 m extremely large telescopes (ELTs) will pave the way for detection and characterization of giant exoplanets down to habitable zone exoplanets around M stars. All three ELT projects eventually have plans for extreme-AO coronagraphs to detect planets (Macintosh et al 2006, Kasper et al 2010, Davies et al 2016). Crossfield (2013) simulates the distribution of Kepler-discovered exoplanets around nearby, very low mass stars and then checked which planets were detectable using extremely large ground-based telescopes with high-contrast imagers and predicts that such a cohort may yield 2-4 directly-imageable planets with radii of 2-4 Earth radii and temperatures of $\sim 300$ $\mathrm{K}$ - in other words, super-Earths in the habitable zone. Quanz et al (2015) simulate performance for the E-ELT facility mid-IR instrument METIS and find that it will be able to image 1) 20 known RV Jovian exoplanets and 2) a handful of superEarths in the habitable zone of nearby M-stars. Combining high-contrast imaging with high-resolution spectroscopy with METIS, Snellen et al (2015) predict that a $R=1.5 R_{\text {earth }}$ planet with a twin-Earth thermal spectrum orbiting $\alpha$ Cen A could be detected in one night of E-ELT time using this method, which also provides the resolution necessary to detect biosignature gas features in the spectrum of such an object. Predicted contrasts for the planned TMT Planet Formation Imager (PFI) as well as other existing and future instruments are presented in Fig. 8

WFIRST-AFTA will be the first custom space coronagraph to directly image exoplanets (Noecker et al 2016). WFIRST-AFTA will directly image old RV planets down to super-Earths in the solar neighborhood, but, with a 2-m mirror diameter, will not have the contrast or resolution to image Earth twins (see Fig. 8 and also Robinson et al (2016)). Robinson et al (2016) simulate expected WFIRST-AFTA coronagraph performance and conclude that WFIRST-AFTA will be able to detect methane absorption in cool Jupiters (Jupiters at 2 AU flux equivalent distance from the Sun) and obtain spectra of cool Neptunes as well as super-Earths. Use of a star-shade with WFIRST-AFTA may eventually enable the detection and characterization of Earth-twins - see e.g. the Exo-S mission concept (Seager et al 2015).

In the longer term future, planned missions such as HabEx and LUVOIR (Dalcanton et al 2015) will combine large diameter space telescopes with state-of-theart-coronagraphs, achieving the $10^{10}$ contrasts and extreme resolutions to detect and characterize low mass planets in the habitable zones around $\mathrm{G}$ stars. In addition, LUVOIR is planned to have a wide wavelength coverage, from IR to UV. As noted previously, as many single biosignature features may also have abiotic sources (e.g. ozone Domagal-Goldman et al 2014; Luger and Barnes 2015; Harman et al 2015), to truly determine if a planet is habitable requires the detection of a constellation of different biosignatures in the planet spectrum, the combination of which prove dis-equilibrium chemistry is present. Additionally, to build a picture of habitability as a function of host star, planet mass, etc., it is vital to characterize habitable zone planets orbiting a wide range of host stars. In Figure 8, contrasts achievable with current and future high resolution imagers are plotted alongside current known directly imaged exoplanets and our own solar system planets (assuming a distance 
of $20 \mathrm{pc}$ ). There are 64 G0-G5 stars within 20 pc of the Earth. While earlier missions and telescopes such as JWST, WFIRST, and the ELTs will yield photometry and spectroscopy of a handful of habitable zone planets around low mass $\mathrm{M}$ and $\mathrm{K}$ dwarfs (e.g. Proxima Centauri b), only a mission like LUVOIR will yield the contrasts and resolutions necessary to detect and characterize true Earth twins (i.e. Earth-sized planets in the habitable zone of Sun-like stars). Through a LUVOIRclass mission we will be able to observe enough planets to reach statistically meaningful conclusions regarding the frequency and characteristics of exo-Earths and better understand our own planet in its astronomical context.

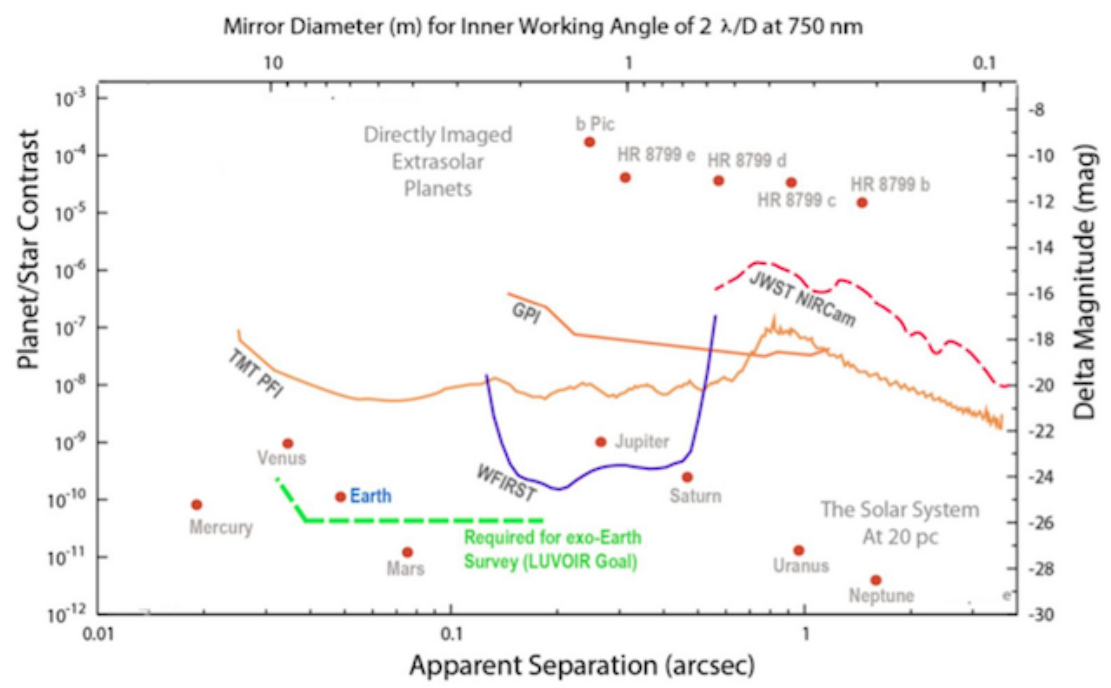

Fig. 8 Contrasts achieved by current exoplanet direct imaging instruments and predicted contrasts for next-generation images, from http://asd.gsfc.nasa.gov/luvoir adapted from Lawson et al (2012); Mawet et al (2012).

\section{Conclusions}

Direct imaging has already transformed our understanding of young giant planets, both as companions and free-floating in young moving groups. Such low-gravity objects have much different atmospheric structures than high-gravity, field brown dwarfs with similar $T_{\text {eff }}$, holding on to thick dusty clouds in their atmospheres and suppressing methane absorption down to much lower temperatures than their higher mass counterparts. Ultimately, current work in direct imaging is a prelude to the era of deep characterization that will begin with JWST and find its ultimate expression in future space telescopes such as LUVOIR. JWST will enable in-depth 
characterization of young giant exoplanets, while the ELTs will allow for the discovery and characterization of old, cold Jupiters as well as habitable zone exoplanets around very nearby M-stars. Eventually, a LUVOIR-class mission will be able to obtain images and spectroscopy of enough habitable zone exo-Earths to reach statistically meaningful conclusions regarding the frequency and characteristics of these planets. Perhaps such a mission will conclusively demonstrate the presence of dis-equilibrium chemistry and show evidence of not just being in the habitable zone but actually being inhabited.

\section{Cross-References}

- Variability of Brown Dwarfs

- Large Scale Searchers for Brown Dwarfs and Free-Floating Planets

- Direct Imaging as an Exoplanet Discovery Method

- Imaging with Adaptive Optics and Coronographs for Exoplanet Research

- SPHERE as an Instrument for Exoplanet Research

- CHARIS on Subaru: An Instrument for Exoplanet Research

- ESPRESSO on VLT: An Instrument for Exoplanet Research

- Space Missions for Exoplanet Science: JWST

- Atmospheric Retrieval for Exoplanet Atmospheres

- Atmospheres as the Window Into Exoplanet Habitability and Life

- HR8799: Imaging a System of Exoplanets

- Future Exoplanet Research: High Contrast Imaging Techniques

Acknowledgements BB gratefully acknowledges support from STFC grant ST/M001229/1.

\section{References}

Allers KN, Gallimore JF, Liu MC Dupuy TJ (2016) The Radial and Rotational Velocities of PSO J318.5338-22.8603, a Newly Confirmed Planetary-mass Member of the $\beta$ Pictoris Moving Group. Astrophys J 819:133, DOI 10.3847/0004-637X/819/2/133, 1601.04717

Amara A Quanz SP (2012) PYNPOINT: an image processing package for finding exoplanets. MNRAS427:948-955, DOI 10.1111/j.1365-2966.2012.21918.x, 1207.6637

Anglada-Escudé G, Amado PJ, Barnes J et al (2016) A terrestrial planet candidate in a temperate orbit around Proxima Centauri. Nature536:437-440, DOI 10.1038/nature19106, 1609. 03449

Apai D, Radigan J, Buenzli E et al (2013) HST Spectral Mapping of L/T Transition Brown Dwarfs Reveals Cloud Thickness Variations. Astrophys J 768:121, DOI 10.1088/0004-637X/768/2/ $121,1303.4151$

Apai D, Kasper M, Skemer A et al (2016) High-cadence, High-contrast Imaging for Exoplanet Mapping: Observations of the HR 8799 Planets with VLT/SPHERE Satellite-spot-corrected Relative Photometry. Astrophys J 820:40, DOI 10.3847/0004-637X/820/1/40, 1602.02856 
Artigau É, Kouach D, Donati JF et al (2014) SPIRou: the near-infrared spectropolarimeter/highprecision velocimeter for the Canada-France-Hawaii telescope. In: Ground-based and Airborne Instrumentation for Astronomy V, Proc SPIE, vol 9147, p 914715, DOI 10.1117/12.2055663, 1406.6992

Atri D (2017) Modelling stellar proton event-induced particle radiation dose on close-in exoplanets. MNRAS465:L34-L38, DOI 10.1093/mnrasl/slw199, 1606.07027

Baraffe I, Chabrier G Gallardo J (2009) Episodic Accretion at Early Stages of Evolution of LowMass Stars and Brown Dwarfs: A Solution for the Observed Luminosity Spread in H-R Diagrams? ApJ702:L27-L31, DOI 10.1088/0004-637X/702/1/L27,0 0907.3886

Barman TS, Macintosh B, Konopacky QM Marois C (2011) Clouds and Chemistry in the Atmosphere of Extrasolar Planet HR8799b. Astrophys J 733:65, DOI 10.1088/0004-637X/733/1/65, 1103.3895

Barnes R, Deitrick R, Luger R et al (2016) The Habitability of Proxima Centauri b I: Evolutionary Scenarios. ArXiv e-prints 1608.06919

Belikov R, Bendek E, Thomas S, Males J Lozi J (2015) How to directly image a habitable planet around Alpha Centauri with a $\sim 30-45 \mathrm{~cm}$ space telescope. In: Techniques and Instrumentation for Detection of Exoplanets VII, Proc SPIE, vol 9605, p 960517, DOI 10.1117/12.2188732, 1510.02479

Benneke B (2015) Strict Upper Limits on the Carbon-to-Oxygen Ratios of Eight Hot Jupiters from Self-Consistent Atmospheric Retrieval. ArXiv e-prints 1504.07655

Beuzit JL, Feldt M, Dohlen K et al (2008) SPHERE: a 'Planet Finder' instrument for the VLT. In: Ground-based and Airborne Instrumentation for Astronomy II, Proc. SPIE, vol 7014, p 701418, DOI 10.1117/12.790120

Biller BA, Close LM, Masciadri E et al (2007) An Imaging Survey for Extrasolar Planets around 45 Close, Young Stars with the Simultaneous Differential Imager at the Very Large Telescope and MMT. ApJS173:143-165, DOI 10.1086/519925

Biller BA, Crossfield IJM, Mancini L et al (2013a) Weather on the Nearest Brown Dwarfs: Resolved Simultaneous Multi-wavelength Variability Monitoring of WISE J104915.57531906.1AB. Astrophys J Lett 778:L10, DOI 10.1088/2041-8205/778/1/L10, 1310.5144

Biller BA, Liu MC, Wahhaj Z et al (2013b) The Gemini/NICI Planet-Finding Campaign: The Frequency of Planets around Young Moving Group Stars. ApJ777:160, DOI 10.1088/0004-637X/ 777/2/160, 1309.1462

Biller BA, Vos J, Bonavita M et al (2015) Variability in a Young, L/T Transition Planetary-mass Object. Astrophys J Lett 813:L23, DOI 10.1088/2041-8205/813/2/L23, 1510.07625

Boccaletti A, Lagage PO, Baudoz P et al (2015) The Mid-Infrared Instrument for the James Webb Space Telescope, V: Predicted Performance of the MIRI Coronagraphs. PASP127:633, DOI $10.1086 / 682256,1508.02352$

Bonnefoy M, Chauvin G, Lagrange AM et al (2014a) A library of near-infrared integral field spectra of young M-L dwarfs. A\&A562:A127, DOI 10.1051/0004-6361/201118270, 1306 . 3709

Bonnefoy M, Marleau GD, Galicher R et al (2014b) Physical and orbital properties of $\beta$ Pictoris b. Astron \& Astrophys 567:L9, DOI 10.1051/0004-6361/201424041, 1407.4001

Bonnefoy M, Zurlo A, Baudino JL et al (2016) First light of the VLT planet finder SPHERE. IV. Physical and chemical properties of the planets around HR8799. Astron \& Astrophys 587:A58, DOI 10.1051/0004-6361/201526906, 1511.04082

Bowler BP, Liu MC, Kraus AL, Mann AW Ireland MJ (2011) A Disk around the Planetary-mass Companion GSC 06214-00210 b: Clues about the Formation of Gas Giants on Wide Orbits. ApJ743:148, DOI 10.1088/0004-637X/743/2/148, 1109.5693

Bowler BP, Liu MC, Kraus AL Mann AW (2014) Spectroscopic Confirmation of Young Planetarymass Companions on Wide Orbits. ApJ784:65, DOI 10.1088/0004-637X/784/1/65, 1401. 7668

Bowler BP, Andrews SM, Kraus AL et al (2015) An ALMA Constraint on the GSC 6214-210 B Circum-Substellar Accretion Disk Mass. ApJ805:L17, DOI 10.1088/2041-8205/805/2/L17, 1505.01483 
Brandt TD, McElwain MW, Turner EL et al (2014) A Statistical Analysis of SEEDS and Other High-contrast Exoplanet Surveys: Massive Planets or Low-mass Brown Dwarfs? ApJ794:159, DOI 10.1088/0004-637X/794/2/159, 1404.5335

Burningham B, Marley MS, Line MR et al (2017) Retrieval of atmospheric properties of cloudy L dwarfs. ArXiv e-prints 1701.01257

Caceres C, Hardy A, Schreiber MR et al (2015) On the Nature of the Tertiary Companion to FW Tau: ALMA CO Observations and SED Modeling. ApJ806:L22, DOI 10.1088/2041-8205/806/ 2/L22, 1504.05644

Cantalloube F, Mouillet D, Mugnier LM et al (2015) Direct exoplanet detection and characterization using the ANDROMEDA method: Performance on VLT/NaCo data. A\&A582:A89, DOI 10.1051/0004-6361/201425571, 1508.06406

Chauvin G, Desidera S, Lagrange AM et al (2017) Discovery of a warm, dusty giant planet around HIP 65426. A\&A605:L9, DOI 10.1051/0004-6361/201731152

Chilcote J, Barman T, Fitzgerald MP et al (2015) The First H-band Spectrum of the Giant Planet $\beta$ Pictoris b. Astrophys J Lett 798:L3, DOI 10.1088/2041-8205/798/1/L3, 1407.4469

Close LM, Males JR, Morzinski K et al (2013) Diffraction-limited Visible Light Images of Orion Trapezium Cluster with the Magellan Adaptive Secondary Adaptive Optics System (MagAO). ApJ774:94, DOI 10.1088/0004-637X/774/2/94, 1308.4155

Close LM, Follette KB, Males JR et al (2014) Discovery of $\mathrm{H} \alpha$ Emission from the Close Companion inside the Gap of Transitional Disk HD 142527. ApJ781:L30, DOI 10.1088/2041-8205/ 781/2/L30, 1401.1273

Crossfield IJM (2013) On high-contrast characterization of nearby, short-period exoplanets with giant segmented-mirror telescopes. A\&A551:A99, DOI 10.1051/0004-6361/201220914, 1301 . 5884

Crossfield IJM (2014) Doppler imaging of exoplanets and brown dwarfs. Astron \& Astrophys 566:A130, DOI 10.1051/0004-6361/201423750, 1404.7853

Crossfield IJM, Biller B, Schlieder JE et al (2014) A global cloud map of the nearest known brown dwarf. Nature 505:654-656, DOI 10.1038/nature12955, 1401.8145

Cushing MC, Kirkpatrick JD, Gelino CR et al (2011) The Discovery of Y Dwarfs using Data from the Wide-field Infrared Survey Explorer (WISE). Astrophys J 743:50, DOI 10.1088/ 0004-637X/743/1/50, 1108.4678

Dalcanton J, Seager S, Aigrain S et al (2015) From Cosmic Birth to Living Earths: The Future of UVOIR Space Astronomy. ArXiv e-prints 1507.04779

Davenport JRA, Kipping DM, Sasselov D, Matthews JM Cameron C (2016) MOST Observations of Our Nearest Neighbor: Flares on Proxima Centauri. ApJ829:L31, DOI 10.3847/2041-8205/ 829/2/L31, 1608.06672

Davies R, Schubert J, Hartl M et al (2016) MICADO: first light imager for the E-ELT. In: Society of Photo-Optical Instrumentation Engineers (SPIE) Conference Series, Proc SPIE, vol 9908, p 99081Z, DOI 10.1117/12.2233047, 1607.01954

De Rosa RJ, Rameau J, Patience J et al (2016) Spectroscopic Characterization of HD 95086 b with the Gemini Planet Imager. Astrophys J 824:121, DOI 10.3847/0004-637X/824/2/121, 1604 . 01411

Domagal-Goldman SD, Segura A, Claire MW, Robinson TD Meadows VS (2014) Abiotic Ozone and Oxygen in Atmospheres Similar to Prebiotic Earth. ApJ792:90, DOI 10.1088/0004-637X/ 792/2/90, 1407.2622

Dorn RJ, Follert R, Bristow P et al (2016) The “+” for CRIRES: enabling better science at infrared wavelength and high spectral resolution at the ESO VLT. In: Society of Photo-Optical Instrumentation Engineers (SPIE) Conference Series, Proc SPIE, vol 9908, p 99080I, DOI $10.1117 / 12.2232837$

Faherty JK, Riedel AR, Cruz KL et al (2016) Population Properties of Brown Dwarf Analogs to Exoplanets. ApJS225:10, DOI 10.3847/0067-0049/225/1/10, 1605.07927

Gagné J, Lafrenière D, Doyon R, Malo L Artigau É (2014) BANYAN. II. Very Low Mass and Substellar Candidate Members to Nearby, Young Kinematic Groups with Previously Known Signs of Youth. Astrophys J 783:121, DOI 10.1088/0004-637X/783/2/121, 1312.5864 
Gagné J, Faherty JK, Cruz KL et al (2015) BANYAN. VII. A New Population of Young Substellar Candidate Members of Nearby Moving Groups from the BASS Survey. ApJS219:33, DOI $10.1088 / 0067-0049 / 219 / 2 / 33,1506.07712$

Gaidos E Williams DM (2004) Seasonality on terrestrial extrasolar planets: inferring obliquity and surface conditions from infrared light curves. New A10:67-77, DOI 10.1016/j.newast.2004.04. 009

Garraffo C, Drake JJ Cohen O (2016) The Space Weather of Proxima Centauri b. ApJ833:L4, DOI 10.3847/2041-8205/833/1/L4, 1609.09076

Gillon M, Triaud AHMJ, Jehin E et al (2013) Fast-evolving weather for the coolest of our two new substellar neighbours. Astron \& Astrophys 555:L5, DOI 10.1051/0004-6361/201321620, 1304.0481

Goldblatt C (2016) Tutorial models of the climate and habitability of Proxima Centauri b: a thin atmosphere is sufficient to distribute heat given low stellar flux. ArXiv e-prints 1608.07263

Groff TD, Chilcote J, Kasdin NJ et al (2016) Laboratory testing and performance verification of the CHARIS integral field spectrograph. In: Society of Photo-Optical Instrumentation Engineers (SPIE) Conference Series, Proc SPIE, vol 9908, p 99080O, DOI 10.1117/12.2233447

Harman CE, Schwieterman EW, Schottelkotte JC Kasting JF (2015) Abiotic $\mathrm{O}_{2}$ Levels on Planets around F, G, K, and M Stars: Possible False Positives for Life? ApJ812:137, DOI 10.1088/ 0004-637X/812/2/137, 1509.07863

Helled R Schubert G (2009) Heavy-element Enrichment of a Jupiter-mass Protoplanet as a Function of Orbital Location. ApJ697:1256-1262, DOI 10.1088/0004-637X/697/2/1256, 0903. 1997

Helling C, Woitke P, Rimmer PB et al (2014) Disk Evolution, Element Abundances and Cloud Properties of Young Gas Giant Planets. Life 4, DOI 10.3390/life4020142, 1403.4420

Ingraham P, Marley MS, Saumon D et al (2014) Gemini Planet Imager Spectroscopy of the HR 8799 Planets c and d. Astrophys J Lett 794:L15, DOI 10.1088/2041-8205/794/1/L15, 1409. 5456

Jenkins JM, Twicken JD, Batalha NM et al (2015) Discovery and Validation of Kepler-452b: A 1.6 R Super Earth Exoplanet in the Habitable Zone of a G2 Star. AJ150:56, DOI 10.1088/ 0004-6256/150/2/56, 1507.06723

Joergens V, Bonnefoy M, Liu Y et al (2013) OTS 44: Disk and accretion at the planetary border. A\&A558:L7, DOI 10.1051/0004-6361/201322432,1310.1936

Kane SR, Hill ML, Kasting JF et al (2016) A Catalog of Kepler Habitable Zone Exoplanet Candidates. ApJ830:1, DOI 10.3847/0004-637X/830/1/1, 1608.00620

Kane SR, Gelino DM Turnbull MC (2017) On the Orbital Inclination of Proxima Centauri b. AJ153:52, DOI 10.3847/1538-3881/153/2/52, 1612.02872

Karalidi T, Apai D, Schneider G, Hanson JR Pasachoff JM (2015) Aeolus: A Markov Chain Monte Carlo Code for Mapping Ultracool Atmospheres. An Application on Jupiter and Brown Dwarf HST Light Curves. Astrophys J 814:65, DOI 10.1088/0004-637X/814/1/65, 1510.04251

Karalidi T, Apai D, Marley MS Buenzli E (2016) Maps of Evolving Cloud Structures in Luhman 16AB from HST Time-resolved Spectroscopy. Astrophys J 825:90, DOI 10.3847/0004-637X/ $825 / 2 / 90,1605.01682$

Kasper M, Beuzit JL, Verinaud C et al (2010) EPICS: direct imaging of exoplanets with the EELT. In: Ground-based and Airborne Instrumentation for Astronomy III, Proc SPIE, vol 7735, pp 77,352E-77,352E-9, DOI 10.1117/12.856850

Kipping DM, Cameron C, Hartman JD et al (2017) No Conclusive Evidence for Transits of Proxima b in MOST Photometry. AJ153:93, DOI 10.3847/1538-3881/153/3/93, 1609.08718

Kirkpatrick JD (2005) New Spectral Types L and T. Ann Rev of Astron \& Astrophys 43:195-245, DOI 10.1146/annurev.astro.42.053102.134017

Kirkpatrick JD, Gelino CR, Cushing MC et al (2012) Further Defining Spectral Type "Y" and Exploring the Low-mass End of the Field Brown Dwarf Mass Function. Astrophys J 753:156, DOI 10.1088/0004-637X/753/2/156, 1205.2122 
Kraus AL, Andrews SM, Bowler BP et al (2015) An ALMA Disk Mass for the Candidate Protoplanetary Companion to FW Tau. ApJ798:L23, DOI 10.1088/2041-8205/798/1/L23, 1412.2175

Kreidberg L Loeb A (2016) Prospects for Characterizing the Atmosphere of Proxima Centauri b. ApJ832:L12, DOI 10.3847/2041-8205/832/1/L12, 1608.07345

Kreidberg L, Bean JL, Désert JM et al (2014a) Clouds in the atmosphere of the super-Earth exoplanet GJ1214b. Nature505:69-72, DOI 10.1038/nature12888, 1401.0022

Kreidberg L, Bean JL, Désert JM et al (2014b) A Precise Water Abundance Measurement for the Hot Jupiter WASP-43b. ApJ793:L27, DOI 10.1088/2041-8205/793/2/L27, 1410.2255

Kuchner MJ Traub WA (2002) A Coronagraph with a Band-limited Mask for Finding Terrestrial Planets. ApJ570:900-908, DOI 10.1086/339625, astro-ph/0203455

Kuzuhara M, Tamura M, Kudo T et al (2013) Direct Imaging of a Cold Jovian Exoplanet in Orbit around the Sun-like Star GJ 504. ApJ774:11, DOI 10.1088/0004-637X/774/1/11, 1307.2886

Lachapelle FR, Lafrenière D, Gagné J et al (2015) Characterization of Low-mass, Wide-separation Substellar Companions to Stars in Upper Scorpius: Near-infrared Photometry and Spectroscopy. ApJ802:61, DOI 10.1088/0004-637X/802/1/61, 1503.07586

Lafrenière D, Marois C, Doyon R, Nadeau D Artigau É (2007) A New Algorithm for Point-Spread Function Subtraction in High-Contrast Imaging: A Demonstration with Angular Differential Imaging. ApJ660:770-780, DOI 10.1086/513180, astro-ph/0702697

Lagrange AM, Bonnefoy M, Chauvin G et al (2010) A Giant Planet Imaged in the Disk of the Young Star $\beta$ Pictoris. Science 329:57, DOI 10.1126/science.1187187, 1006.3314

Lavie B, Mendonça JM, Mordasini C et al (2016) HELIOS-Retrieval: An Open-source, Nested Sampling Atmospheric Retrieval Code, Application to the HR 8799 Exoplanets and Inferred Constraints for Planet Formation. ArXiv e-prints 1610.03216

Lavigne JF, Doyon R, Lafrenière D, Marois C Barman T (2009) Near-Infrared Observations of GQ Lup b Using the Gemini Integral Field Spectrograph NIFS. ApJ704:1098-1106, DOI 10.1088/0004-637X/704/2/1098, 0908.3723

Lawson PR, Poyneer L, Barrett $\mathrm{H}$ et al (2012) On advanced estimation techniques for exoplanet detection and characterization using ground-based coronagraphs. In: Adaptive Optics Systems III, Proc SPIE, vol 8447, p 844722, DOI 10.1117/12.925099

Lee JM, Heng K Irwin PGJ (2013) Atmospheric Retrieval Analysis of the Directly Imaged Exoplanet HR 8799b. ApJ778:97, DOI 10.1088/0004-637X/778/2/97, 1307.1404

Lew BWP, Apai D, Zhou Y et al (2016) Cloud Atlas: Discovery of Patchy Clouds and Highamplitude Rotational Modulations in a Young, Extremely Red L-type Brown Dwarf. Astrophys J Lett 829:L32, DOI 10.3847/2041-8205/829/2/L32, 1609.04804

Line MR, Fortney JJ, Marley MS Sorahana S (2014) A Data-driven Approach for Retrieving Temperatures and Abundances in Brown Dwarf Atmospheres. ApJ793:33, DOI 10.1088/ 0004-637X/793/1/33, 1403.6412

Line MR, Teske J, Burningham B, Fortney JJ Marley MS (2015) Uniform Atmospheric Retrieval Analysis of Ultracool Dwarfs. I. Characterizing Benchmarks, G1 570D and HD 3651B. ApJ807:183, DOI 10.1088/0004-637X/807/2/183, 1504.06670

Liu MC, Magnier EA, Deacon NR et al (2013) The Extremely Red, Young L Dwarf PSO J318.5338-22.8603: A Free-floating Planetary-mass Analog to Directly Imaged Young Gasgiant Planets. Astrophys J Lett 777:L20, DOI 10.1088/2041-8205/777/2/L20, 1310 . 0457

Lovis C, Snellen I, Mouillet D et al (2016) Atmospheric characterization of Proxima b by coupling the Sphere high-contrast imager to the Espresso spectrograph. ArXiv e-prints 1609.03082

Luger R Barnes R (2015) Extreme Water Loss and Abiotic O2Buildup on Planets Throughout the Habitable Zones of M Dwarfs. Astrobiology 15:119-143, DOI 10.1089/ast.2014.1231, 1411. 7412

Luger R, Lustig-Yaeger J, Fleming DP et al (2016) The Pale Green Dot: A Method to Characterize Proxima Centauri b using Exo-Aurorae. ArXiv e-prints 1609.09075

Luhman KL (2013) Discovery of a Binary Brown Dwarf at 2 pc from the Sun. Astrophys J Lett 767:L1, DOI 10.1088/2041-8205/767/1/L1, 1303.2401 
Luhman KL (2014) Discovery of a $250 \mathrm{~K}$ Brown Dwarf at 2 pc from the Sun. Astrophys J Lett 786:L18, DOI 10.1088/2041-8205/786/2/L18, 1404.6501

Luhman KL, Patten BM, Marengo M et al (2007) Discovery of Two T Dwarf Companions with the Spitzer Space Telescope. ApJ654:570-579, DOI 10.1086/509073, astro-ph/0609464

MacGregor MA, Wilner DJ, Czekala I et al (2017) ALMA Measurements of Circumstellar Material in the GQ Lup System. ApJ835:17, DOI 10.3847/1538-4357/835/1/17, 1611.06229

Macintosh B, Troy M, Doyon R et al (2006) Extreme adaptive optics for the Thirty Meter Telescope. In: Society of Photo-Optical Instrumentation Engineers (SPIE) Conference Series, Proc SPIE, vol 6272, p 62720N, DOI 10.1117/12.672032

Macintosh B, Graham JR, Ingraham P et al (2014) First light of the Gemini Planet Imager. Proceedings of the National Academy of Science 111:12,661-12,666, DOI 10.1073/pnas.1304215111, 1403.7520

Macintosh B, Graham JR, Barman T et al (2015) Discovery and spectroscopy of the young jovian planet 51 Eri b with the Gemini Planet Imager. Science 350:64-67, DOI 10.1126/science. aac5891, 1508.03084

Madhusudhan N, Burrows A Currie T (2011) Model Atmospheres for Massive Gas Giants with Thick Clouds: Application to the HR 8799 Planets and Predictions for Future Detections. ApJ737:34, DOI 10.1088/0004-637X/737/1/34, 1102.5089

Maire AL, Bonnefoy M, Ginski C et al (2016) First light of the VLT planet finder SPHERE. II. The physical properties and the architecture of the young systems PZ Telescopii and HD 1160 revisited. A\&A587:A56, DOI 10.1051/0004-6361/201526594, 1511.04072

Marley MS, Fortney JJ, Hubickyj O, Bodenheimer P Lissauer JJ (2007) On the Luminosity of Young Jupiters. ApJ655:541-549, DOI 10.1086/509759, astro-ph/0609739

Marois C, Lafrenière D, Doyon R, Macintosh B Nadeau D (2006) Angular Differential Imaging: A Powerful High-Contrast Imaging Technique. ApJ641:556-564, DOI 10.1086/500401, astro-ph/0512335

Marois C, Macintosh B, Barman T et al (2008) Direct Imaging of Multiple Planets Orbiting the Star HR 8799. Science 322:1348, DOI 10.1126/science.1166585, 0811.2606

Marois C, Zuckerman B, Konopacky QM, Macintosh B Barman T (2010) Images of a fourth planet orbiting HR 8799. Nature468:1080-1083, DOI 10.1038/nature09684, 1011.4918

Maurin AS, Selsis F, Hersant F Belu A (2012) Thermal phase curves of nontransiting terrestrial exoplanets. II. Characterizing airless planets. A\&A538:A95, DOI 10.1051/0004-6361/ 201117054, 1110.3087

Mawet D, Pueyo L, Lawson P et al (2012) Review of small-angle coronagraphic techniques in the wake of ground-based second-generation adaptive optics systems. In: Space Telescopes and Instrumentation 2012: Optical, Infrared, and Millimeter Wave, Proc SPIE, vol 8442, p 844204, DOI 10.1117/12.927245, 1207.5481

Meadows VS, Arney GN, Schwieterman EW et al (2016) The Habitability of Proxima Centauri b: II: Environmental States and Observational Discriminants. ArXiv e-prints 1608.08620

Metchev SA, Heinze A, Apai D et al (2015) Weather on Other Worlds. II. Survey Results: Spots are Ubiquitous on L and T Dwarfs. Astrophys J 799:154, DOI 10.1088/0004-637X/799/2/154, 1411.3051

Mordasini C, Alibert Y Benz W (2009) Extrasolar planet population synthesis. I. Method, formation tracks, and mass-distance distribution. A\&A501:1139-1160, DOI 10.1051/0004-6361/ 200810301,0904.2524

Morley CV, Fortney JJ, Marley MS et al (2015) Thermal Emission and Reflected Light Spectra of Super Earths with Flat Transmission Spectra. ApJ815:110, DOI 10.1088/0004-637X/815/2/ $110,1511.01492$

Morzinski KM, Males JR, Skemer AJ et al (2015) Magellan Adaptive Optics First-light Observations of the Exoplanet $\beta$ Pic b. II. 3-5 $\mu \mathrm{m}$ Direct Imaging with $\mathrm{MagAO}+\mathrm{Clio}$, and the Empirical Bolometric Luminosity of a Self-luminous Giant Planet. ApJ815:108, DOI 10.1088/0004-637X/815/2/108, 1511.02894

Natta A, Testi L, Muzerolle J et al (2004) Accretion in brown dwarfs: An infrared view. A\&A424:603-612, DOI 10.1051/0004-6361:20040356, astro-ph/0406106 
Naud ME, Artigau É, Malo L et al (2014) Discovery of a Wide Planetary-mass Companion to the Young M3 Star GU Psc. ApJ787:5, DOI 10.1088/0004-637X/787/1/5, 1405.2932

Noecker MC, Zhao F, Demers R et al (2016) Coronagraph instrument for WFIRST-AFTA. Journal of Astronomical Telescopes, Instruments, and Systems 2(1):011001, DOI 10.1117/1.JATIS.2. 1.011001

Öberg KI Bergin EA (2016) Excess C/O and C/H in Outer Protoplanetary Disk Gas. ApJ831:L19, DOI 10.3847/2041-8205/831/2/L19, 1610.07859

Öberg KI, Murray-Clay R Bergin EA (2011) The Effects of Snowlines on C/O in Planetary Atmospheres. ApJ743:L16, DOI 10.1088/2041-8205/743/1/L16, 1110.5567

O'Malley-James JT Kaltenegger L (2016) Biofluorescent Worlds: Biological fluorescence as a temporal biosignature for flare star worlds. ArXiv e-prints 1608.06930

Oppenheimer BR, Baranec C, Beichman C et al (2013) Reconnaissance of the HR 8799 Exosolar System. I. Near-infrared Spectroscopy. ApJ768:24, DOI 10.1088/0004-637X/768/1/24, 1303.2627

Quanz SP, Crossfield I, Meyer MR, Schmalzl E Held J (2015) Direct detection of exoplanets in the 3-10 $\mu \mathrm{m}$ range with E-ELT/METIS. International Journal of Astrobiology 14:279-289, DOI 10.1017/S1473550414000135, 1404.0831

Quillen AC Trilling DE (1998) Do Proto-jovian Planets Drive Outflows? ApJ508:707-713, DOI 10.1086/306421, astro-ph/9712033

Quirrenbach A, Amado PJ, Caballero JA et al (2016) CARMENES: an overview six months after first light. In: Society of Photo-Optical Instrumentation Engineers (SPIE) Conference Series, Proc SPIE, vol 9908, p 990812, DOI 10.1117/12.2231880

Racine R, Walker GAH, Nadeau D, Doyon R Marois C (1999) Speckle Noise and the Detection of Faint Companions. PASP111:587-594, DOI 10.1086/316367

Radigan J (2014) An Independent Analysis of the Brown Dwarf Atmosphere Monitoring (BAM) Data: Large-amplitude Variability is Rare Outside the L/T Transition. Astrophys J 797:120, DOI 10.1088/0004-637X/797/2/120, 1408.5919

Radigan J, Lafrenière D, Jayawardhana R Artigau E (2014) Strong Brightness Variations Signal Cloudy-to-clear Transition of Brown Dwarfs. Astrophys J 793:75, DOI 10.1088/0004-637X/ 793/2/75, 1404.3247

Rameau J, Chauvin G, Lagrange AM et al (2013) Discovery of a Probable 4-5 Jupiter-mass Exoplanet to HD 95086 by Direct Imaging. ApJ772:L15, DOI 10.1088/2041-8205/772/2/L15, 1305.7428

Riaud P Schneider J (2007) Improving Earth-like planets' detection with an ELT: the differential radial velocity experiment. A\&A469:355-361, DOI 10.1051/0004-6361:20077085

Ribas I, Bolmont E, Selsis F et al (2016) The habitability of Proxima Centauri b. I. Irradiation, rotation and volatile inventory from formation to the present. A\&A596:A111, DOI 10.1051/ 0004-6361/201629576, 1608.06813

Ricker GR, Winn JN, Vanderspek R et al (2014) Transiting Exoplanet Survey Satellite (TESS). In: Space Telescopes and Instrumentation 2014: Optical, Infrared, and Millimeter Wave, Proc SPIE, vol 9143, p 914320, DOI 10.1117/12.2063489, 1406.0151

Robinson TD, Stapelfeldt KR Marley MS (2016) Characterizing Rocky and Gaseous Exoplanets with 2 m Class Space-based Coronagraphs. PASP128(2):025,003, DOI 10.1088/1538-3873/ $128 / 960 / 025003,1507.00777$

Sallum S, Follette KB, Eisner JA et al (2015) Accreting protoplanets in the LkCa 15 transition disk. Nature 527:342-344, DOI 10.1038/nature15761,1511.07456

Samland M, Mollière P, Bonnefoy M et al (2017) Spectral and atmospheric characterization of 51 Eridani b using VLT/SPHERE. A\&A603:A57, DOI 10.1051/0004-6361/201629767

Schlieder JE, Lépine S Simon M (2012) Likely Members of the $\beta$ Pictoris and AB Doradus Moving Groups in the North. AJ144:109, DOI 10.1088/0004-6256/144/4/109, 1208.0679

Schmidt TOB, Neuhäuser R, Seifahrt A et al (2008) Direct evidence of a sub-stellar companion around CT Chamaeleontis. A\&A491:311-320, DOI 10.1051/0004-6361:20078840, 0809.2812 
Schwarz H, Ginski C, de Kok RJ et al (2016) The slow spin of the young sub-stellar companion GQ Lupi $b$ and its orbital configuration. ArXiv e-prints 1607.00012

Seager S Deming D (2009) On the Method to Infer an Atmosphere on a Tidally Locked Super Earth Exoplanet and Upper Limits to GJ 876d. ApJ703:1884-1889, DOI 10.1088/0004-637X/ 703/2/1884,0910.1505

Seager S, Turnbull M, Sparks W et al (2015) The Exo-S probe class starshade mission. In: Techniques and Instrumentation for Detection of Exoplanets VII, Proc SPIE, vol 9605, p 96050W, DOI 10.1117/12.2190378

Seifahrt A, Neuhäuser R Hauschildt PH (2007) Near-infrared integral-field spectroscopy of the companion to GQ Lupi. A\&A463:309-313, DOI 10.1051/0004-6361:20066463, astro-ph/ 0612250

Selsis F, Wordsworth RD Forget F (2011) Thermal phase curves of nontransiting terrestrial exoplanets. I. Characterizing atmospheres. A\&A532:A1, DOI 10.1051/0004-6361/201116654, 1104.4763

Selsis F, Maurin AS, Hersant F et al (2013) The effect of rotation and tidal heating on the thermal lightcurves of super Mercuries. A\&A555:A51, DOI 10.1051/0004-6361/201321661, 1305. 3858

Shields AL, Ballard S Johnson JA (2016) The Habitability of Planets Orbiting M-dwarf Stars. ArXiv e-prints 1610.05765

Sing DK, Fortney JJ, Nikolov N et al (2016) A continuum from clear to cloudy hot-Jupiter exoplanets without primordial water depletion. Nature529:59-62, DOI 10.1038/nature16068, 1512.04341

Skemer AJ, Hinz PM, Esposito S et al (2012) First Light LBT AO Images of HR 8799 bcde at 1.6 and $3.3 \mu \mathrm{m}$ : New Discrepancies between Young Planets and Old Brown Dwarfs. Astrophys J 753:14, DOI 10.1088/0004-637X/753/1/14, 1203.2615

Skemer AJ, Marley MS, Hinz PM et al (2014) Directly Imaged L-T Transition Exoplanets in the Mid-infrared. Astrophys J 792:17, DOI 10.1088/0004-637X/792/1/17, 1311.2085

Skemer AJ, Morley CV, Allers KN et al (2016) The First Spectrum of the Coldest Brown Dwarf. ApJ826:L17, DOI 10.3847/2041-8205/826/2/L17, 1605.04902

Snellen I, de Kok R, Birkby JL et al (2015) Combining high-dispersion spectroscopy with high contrast imaging: Probing rocky planets around our nearest neighbors. A\&A576:A59, DOI 10.1051/0004-6361/201425018, 1503.01136

Snellen IAG, Brandl BR, de Kok RJ et al (2014) Fast spin of the young extrasolar planet $\beta$ Pictoris b. Nature 509:63-65, DOI 10.1038/nature13253

Soummer R, Pueyo L Larkin J (2012) Detection and Characterization of Exoplanets and Disks Using Projections on Karhunen-Loève Eigenimages. ApJ755:L28, DOI 10.1088/2041-8205/ 755/2/L28, 1207.4197

Sullivan PW, Winn JN, Berta-Thompson ZK et al (2015) The Transiting Exoplanet Survey Satellite: Simulations of Planet Detections and Astrophysical False Positives. ApJ809:77, DOI 10.1088/0004-637X/809/1/77, 1506.03845

Trauger JT Traub WA (2007) A laboratory demonstration of the capability to image an Earth-like extrasolar planet. Nature446:771-773, DOI 10.1038/nature05729

Tremblin P, Amundsen DS, Mourier P et al (2015) Fingering Convection and Cloudless Models for Cool Brown Dwarf Atmospheres. Astrophys J Lett 804:L17, DOI 10.1088/2041-8205/804/ 1/L17, 1504.03334

Tremblin P, Amundsen DS, Chabrier G et al (2016) Cloudless Atmospheres for L/T Dwarfs and Extrasolar Giant Planets. Astrophys J Lett 817:L19, DOI 10.3847/2041-8205/817/2/L19, 1601.03652

Turbet M, Leconte J, Selsis F et al (2016) The habitability of Proxima Centauri b. II. Possible climates and observability. A\&A596:A112, DOI 10.1051/0004-6361/201629577, 1608 . 06827

Vigan A, Langlois M, Moutou C Dohlen K (2008) Exoplanet characterization with long slit spectroscopy. A\&A489:1345-1354, DOI 10.1051/0004-6361:200810090,0808.3817 
Wilson PA, Rajan A Patience J (2014) The brown dwarf atmosphere monitoring (BAM) project. I. The largest near-IR monitoring survey of L and T dwarfs. Astron \& Astrophys 566:A111, DOI 10.1051/0004-6361/201322995, 1404.4633

Wu YL, Sheehan PD, Males JR et al (2017) An ALMA and MagAO Study of the Substellar Companion GQ Lup B. ArXiv e-prints 1701.07541

Yang H, Apai D, Marley MS et al (2016) Extrasolar Storms: Pressure-dependent Changes in Lightcurve Phase in Brown Dwarfs from Simultaneous HST and Spitzer Observations. Astrophys J 826:8, DOI 10.3847/0004-637X/826/1/8, 1605.02708

Yates JS, Palmer PI, Biller B Cockell CS (2016) Atmospheric Habitable Zones in Cool Y Dwarf Atmospheres. ArXiv e-prints 1611.09074

Zapatero Osorio MR, Martín EL, Bouy H et al (2006) Spectroscopic Rotational Velocities of Brown Dwarfs. Astrophys J 647:1405-1412, DOI 10.1086/505484, astro-ph/0603194

Zhou Y, Herczeg GJ, Kraus AL, Metchev S Cruz KL (2014) Accretion onto Planetary Mass Companions of Low-mass Young Stars. ApJ783:L17, DOI 10.1088/2041-8205/783/1/L17, 1401.6545

Zhou Y, Apai D, Schneider GH, Marley MS Showman AP (2016) Discovery of Rotational Modulations in the Planetary-mass Companion 2M1207b: Intermediate Rotation Period and Heterogeneous Clouds in a Low Gravity Atmosphere. Astrophys J 818:176, DOI 10.3847/0004-637X/ 818/2/176, 1512.02706

Zuckerman B Song I (2004) Young Stars Near the Sun. ARA\&A42:685-721, DOI 10.1146/ annurev.astro.42.053102.134111

Zurlo A, Vigan A, Galicher R et al (2016) First light of the VLT planet finder SPHERE. III. New spectrophotometry and astrometry of the HR 8799 exoplanetary system. Astron \& Astrophys 587:A57, DOI 10.1051/0004-6361/201526835, 1511.04083 